\title{
SECOND ORDER OPTIMALITY CONDITIONS FOR PERIODIC OPTIMAL CONTROL PROBLEMS GOVERNED BY SEMILINEAR PARABOLIC DIFFERENTIAL EQUATIONS*
}

\author{
HANBIng LiU $^{1, * *}$ AND Gengsheng WANG ${ }^{2}$
}

\begin{abstract}
In this paper, we study second-order optimality conditions for some optimal control problems governed by some semi-linear parabolic equations with periodic state constraint in time. We obtain a necessary condition and a sufficient condition in terms of the second order derivative of the associated Lagrangian. These two conditions correspond to the positive definite and the nonnegativity of the second order derivative of the Lagrangian on the same cone, respectively.
\end{abstract}

Mathematics Subject Classification. 49K20, 35B10, 90C48.

Received January 14, 2020. Accepted March 10, 2021.

\section{INTRODUCTION}

Notation: Given a Banach space $X$, we use $X^{*}$ and $\|\cdot\|_{X}$ to denote its dual space and its norm respectively; use $\langle\cdot, \cdot\rangle_{X}$ to denote its inner product when it is a Hilbert space; and use $\langle\cdot, \cdot\rangle_{X^{*}, X}$ to denote the paring between $X^{*}$ and $X$. Given two linear normed spaces $X_{1}$ and $X_{2}$, we use $X_{1} \hookrightarrow X_{2}$ to denote that $X_{1}$ is continuously imbedded in $X_{2}$, and use $L\left(X_{1} ; X_{2}\right)$ to denote the space of linear continuous operators from $X_{1}$ to $X_{2}$. Let $\Omega \subset \mathbb{R}^{N}$ (with $N=1,2,3)$ be a bounded domain with a $C^{2}$ boundary $\partial \Omega$. Let $\omega \subset \Omega$ be an open and nonempty subset with its characteristic function $m$. For the space $\mathbb{R}^{N}$, we denote by $\|\cdot\|_{N}$ its Euclidean norm. Given matrix $D$, we use $D^{\top}$ to denote the transpose of $D$. Write $H \triangleq L^{2}(\Omega)$. Define $A: H \rightarrow H$, with $D(A)=H^{2}(\Omega) \cap H_{0}^{1}(\Omega)$, by $A y \triangleq-\sum_{i, j=1}^{N}\left[a_{i j}(x) y_{x_{i}}\right]_{x_{j}}, y \in D(A)$, where $a_{i j}(\cdot) \in C^{2}(\bar{\Omega})$ satisfies that $a_{i j}(x)=a_{j i}(x)$ for all $x \in \Omega$ and $i, j=1, \ldots, N$ and that for some $\Lambda>0$,

$$
\sum_{i, j=1}^{N} a_{i j} \xi_{i} \xi_{j} \geq \Lambda\|\xi\|_{N}^{2} \text { for all } \xi \in \mathbb{R}^{N}
$$

* This work was Supported by the National Natural Science Foundation of China under grant No. 11971022 and No. 11926337, and the Fundamental Research Funds for the Central Universities, China University of Geosciences (Wuhan) under grant CUGSX01.

Keywords and phrases: Semi-linear parabolic equations, periodic state constraint, second order optimality conditions.

1 School of Mathematics and Physics, China University of Geosciences (Wuhan), Wuhan 430074, P.R. China.

2 Center for Applied Mathematics, Tianjin University, Tianjin 300072, P.R. China.

** Corresponding author: hanbing272003@aliyun.com 
Write $V \triangleq H_{0}^{1}(\Omega)$ with its dual $V^{*} \triangleq H^{-1}(\Omega)$. (Notice that $A$ can be extended to a symmetric, bounded and coercive operator from $V$ to $V^{*}$ by a standard way.) Write $Y \triangleq W^{1,2}([0, T] ; H) \cap L^{2}(0, T ; D(A))$, with the norm: $\|y\|_{Y}=\left(\|y\|_{W^{1,2}([0, T] ; H)}^{2}+\|y\|_{L^{2}(0, T ; D(A))}^{2}\right)^{\frac{1}{2}}, y \in Y$. It is known that $Y \hookrightarrow C([0, T] ; V)$ (see [31]).

Problem: Arbitrarily fix $T>0$ and write $Q \triangleq(0, T) \times \Omega$. We will study second order optimality conditions for the optimal control problem:

$$
(P) \quad \inf _{(y, u) \in Y \times L^{2}(Q)} J(y, u) \triangleq \inf _{(y, u) \in Y \times L^{2}(Q)} \int_{Q}[g(t, y)+h(u)] \mathrm{d} x \mathrm{~d} t
$$

subject to

$$
\begin{gathered}
y^{\prime}(t)+A y(t)+f(t, y)=m u(t), \quad t \in(0, T) ; \\
y(0)=y(T),
\end{gathered}
$$

under some assumptions (on $f, g$ and $h$, which will be given later ). Several concepts and notes on the problem $(P)$ are given in order.

$\left(\mathbf{a}_{1}\right)$ A pair $(y, u) \in Y \times L^{2}(Q)$ is called admissible or an admissible pair, if it satisfies (1.3)-(1.4). The set of all admissible pairs will be denoted by $\mathcal{P}_{a d}$. Thus, the problem $(P)$ turns to be the problem: $\inf _{(y, u) \in \mathcal{P}_{a d}} J(y, u)$.

$\left(\mathbf{a}_{2}\right)$ An admissible pair $(\widetilde{y}, \widetilde{u})$ is called a global minimizer (or a minimizer for simplicity), if $J(\widetilde{y}, \widetilde{u}) \leq J(y, u)$ for all $(y, u) \in \mathcal{P}_{a d}$.

$\left(\mathbf{a}_{3}\right)$ For each $(\hat{y}, \hat{u}) \in \mathcal{P}_{a d}$ and each $\delta>0$, we define

$$
\mathcal{B}_{\delta}(\hat{y}, \hat{u})=\left\{(y, u) \in \mathcal{P}_{a d}:\|y-\hat{y}\|_{Y} \leq \delta,\|u-\hat{u}\|_{L^{2}(Q)} \leq \delta\right\} .
$$

An admissible pair $\left(y^{*}, u^{*}\right)$ is called a weak local minimizer, if there exists $\delta>0$ so that $J\left(y^{*}, u^{*}\right) \leq J(y, u)$ for all $(y, u) \in \mathcal{B}_{\delta}\left(y^{*}, u^{*}\right)$.

$\left(\mathbf{a}_{4}\right)$ An admissible pair $\left(y^{*}, u^{*}\right)$ is called a normal extremal, if there is $p^{*} \in Y$ so that

$$
\left\{\begin{array}{l}
-p^{* \prime}(t)+A p^{*}(t)+f_{y}\left(t, y^{*}\right) p^{*}(t)=-g_{y}\left(t, y^{*}\right), \text { a.e. } t \in(0, T), \\
p^{*}(0)=p^{*}(T),
\end{array}\right.
$$

and

$$
m p^{*}(t)=h^{\prime}\left(u^{*}(t)\right) \text {, a.e. } t \in(0, T) .
$$

The above $p^{*} \in Y$ is called the costate associated to $\left(y^{*}, u^{*}\right)$. When $\left(y^{*}, u^{*}\right)$ is a normal extremal, the costate is unique (see Thm. 2.1). Notice that (1.7) is a consequence of the well known Pontryagin's maximum condition.

$\left(\mathbf{a}_{5}\right)$ It has been shown in [42] that for the problem $(P)$, any global minimizer is a normal extremal.

Assumptions: The following assumptions will be effective throughout the paper:

(H1) The function $f: \mathbb{R}^{+} \times \mathbb{R} \rightarrow \mathbb{R}$ is continuous and for each $t \in[0, T], f(t, \cdot)$ is of class $C^{2}$. Further, there is $C>0$ such that

$$
\begin{aligned}
& \left|f_{r}(t, r)\right| \leq C\left(|r|^{r_{1}}+1\right) \text { for all } t \in \mathbb{R}^{+} \text {and } r \in \mathbb{R} ; \\
& \left|f_{r r}(t, r)\right| \leq C\left(|r|^{r_{2}}+1\right) \text { for all } t \in \mathbb{R}^{+} \text {and } r \in \mathbb{R}
\end{aligned}
$$




$$
\left|f_{r r}(t, r)-f_{r r}(t, \hat{r})\right| \leq C\left(1+|r|^{r_{3}}+|\hat{r}|^{r_{3}}\right)|r-\hat{r}| \text { for all } t \in[0, T] \text { and } r, \hat{r} \in \mathbb{R} \text {. }
$$

Here, $r_{1}, r_{2}, r_{3} \geq 0$ when $N=1,2$, while $0 \leq r_{1} \leq 2,0 \leq r_{2} \leq 1, r_{3}=0$, when $N=3$.

(H2) The function $g:[0, T] \times \mathbb{R} \rightarrow \mathbb{R}^{+}$is continuous and $g(t, \cdot)$ is of class $C^{2}$ for each $t \in[0, T]$. Further, there is $C>0$ such that

$$
\begin{aligned}
& \left|g_{r}(t, r)\right| \leq C\left(|r|^{m_{1}}+1\right) \text { for all } t \in \mathbb{R}^{+} \text {and } r \in \mathbb{R} ; \\
& \left|g_{r r}(t, r)\right| \leq C\left(|r|^{m_{2}}+1\right) \text { for all } t \in \mathbb{R}^{+} \text {and } r \in \mathbb{R} ; \\
& \left|g_{r r}(t, r)-g_{r r}(t, \hat{r})\right| \leq C\left(1+|r|^{m_{3}}+|\hat{r}|^{m_{3}}\right)|r-\hat{r}| \text { for all } t \in[0, T] \text { and } r, \hat{r} \in \mathbb{R} .
\end{aligned}
$$

Here, $m_{1}, m_{2}, m_{3} \geq 0$ when $N=1,2$, while $0 \leq m_{1} \leq 3,0 \leq m_{2} \leq 2,0 \leq m_{3} \leq 1$, when $N=3$.

(H3) The function $h: \mathbb{R} \rightarrow(-\infty,+\infty]$ is convex quadratic, i.e., there is a positive constant $\alpha$ such that $h^{\prime \prime}(r)=\alpha$ for all $r \in \mathbb{R}$.

About the above assumptions, we give several notes.

$\left(\mathbf{b}_{1}\right)$ In general, the state equation (1.3) with $f$ satisfying (H1) may have no solution over the given interval $[0, T]$. A typical example on $f$ with $(H 1)$ is as: $f(r)=-|r|^{\gamma-1} r, r \in \mathbb{R}$, where $\gamma>1$ when $N=1,2$, and $1<\gamma \leq 3$ when $N=3$. In this case, it is known that, the solution to state equation (1.3), with null control and an initial condition $y(0)=y_{0} \in V$, has a unique solution $y \in C\left(\left[0, t_{\max }\right) ; V\right)$ on a maximal interval of existence $\left[0, t_{\max }\right)$, and $t_{\max }$ depends on the initial value $y_{0}$ (see Thm. 3.2 in [3]). When the control is not zero, the blow up problem has been studied in [28] (see Prop. 2.2 in [28]). It is possible that $t_{\max }<T$ and the solution does not exist over the given interval $[0, T]$. The problem $(P)$, with this type of $f$, is referred to as a non-well-posed optimal control problem (see Sect. 4.1, Chap. 7 in [30], where optimal control problem of (1.3) was studied in the case $\left.f(r)=-r^{3}\right)$.

$\left(\mathbf{b}_{2}\right)$ We give a specific example here that all the assumptions listed above are fulfilled.

$$
(P) \inf _{(y, u) \in Y \times L^{2}(Q)} J(y, u) \triangleq \inf _{(y, u) \in Y \times L^{2}(Q)} \int_{Q}\left(|y|^{2}+|u|^{2}\right) \mathrm{d} x \mathrm{~d} t
$$

subject to

$$
y^{\prime}(t)+A y(t)-y^{3}(t)-8 \pi^{3} \cos y(t)=m u(t), \quad t \in(0, T) ; \quad y(0)=y(T)
$$

We can see that when $u=0, \underline{y}=-2 \pi$ is a subsolution and $\bar{y}=\pi$ is a supersolution. By Theorem 1.2 in [2], this problem has at least one nontrivial admissible pair $(y, 0)$.

$\left(\mathbf{b}_{3}\right)$ The conditions on $f$ and $g$ given in $(H 1)$ and $(H 2)$ ensure some estimates on nonlinear or linearized terms in the problem $(P)$. These estimates are technically important in proofs of our main results.

$\left(\mathbf{b}_{4}\right)$ Since our control space is $L^{2}(Q)$, we can get a qualified first order necessary condition (without using $(H 3)$ ). Based on the qualified first order necessary condition, and with aid of (H3), we are able to get the second order optimality conditions. Usually, the condition $(H 3)$ can be relaxed if one choose control space as $L^{\infty}(Q)$, but in this case we do not know how to get a qualified first order necessary condition for our problem $(P)$. (At least, the method used in this paper does not work.)

Main results: The main results of this paper are as follows:

Second order sufficient optimality condition: Let $\left(y^{*}, u^{*}\right)$ be a normal extremal of $(P)$, and let $p^{*}$ be the associated costate. If there is $\gamma>0$ such that the second order derivative of the Lagrangian function $\mathcal{L}: Y \times L^{2}(Q) \rightarrow \mathbb{R}$, 
which is defined by

$$
\mathcal{L}(y, u)=J(y, u)+\int_{0}^{T} \int_{\Omega} p^{*}\left(y^{\prime}(t)+A y+f(t, y)-m u\right) \mathrm{d} x \mathrm{~d} t
$$

satisfies the following positive definite condition at $\left(y^{*}, u^{*}\right)$ :

$$
\mathcal{L}^{\prime \prime}\left(y^{*}, u^{*}\right)(z, v)^{2} \geq \gamma\left(\|z(0)\|_{H}^{2}+\|v\|_{L^{2}(Q)}^{2}\right)
$$

for all $(z, v)$ in the critical cone:

$$
\begin{aligned}
\mathcal{C}\left(y^{*}, u^{*}\right) \triangleq & \left\{(z, v) \in Y \times L^{2}(Q):\right. \\
& \left.z^{\prime}(t)+A z(t)+f_{y}\left(t, y^{*}\right) z(t)=m v(t), t \in(0, T), \quad z(0)=z(T)\right\},
\end{aligned}
$$

then, $\left(y^{*}, u^{*}\right)$ is a weak local minimizer for problem $(P)$.

The second order necessary optimality conditions: Let $\left(y^{*}, u^{*}\right)$ be a weak local minimizer for the problem $(P)$. Then, it is a normal extremal. Furthermore, if $p^{*}$ is the associated costate, then, the second order derivative of the Lagrangian function $\mathcal{L}$ at $\left(y^{*}, u^{*}\right)$ satisfies

$$
\mathcal{L}^{\prime \prime}\left(y^{*}, u^{*}\right)(z, v)^{2} \geq 0 \text { for all }(z, v) \in \mathcal{C}\left(y^{*}, u^{*}\right) .
$$

The above results will be restated in Theorems 3.2 and 4.1, as well as Theorem 2.1. We give two notes here.

$\left(\mathbf{c}_{1}\right)$ We consider the second order optimality conditions only for weak local minimizers. There are a few results on the second order optimality conditions for strong local minimizers of elliptic or parabolic optimal control problems, for which we mention $[7,8,18]$. The approaches in theses works rely basically on the well-posedness of the state equation. It would be interesting to investigate the second order optimality conditions for strong local minima of non-well-posed optimal control problems. We think of that when studying them, new techniques might be developed.

$\left(\mathbf{c}_{2}\right)$ It seems that we can extend the methodology in this work to obtain a type of sufficient condition when the box-constraints are imposed on the control, and the critical cone in this case involves strongly active constraint. For this purpose, we should preliminarily impose a regularity assumption on the local extremal (see [16]). In a more concrete form, we can assume that the intersection of the set $\omega$ and the set of points at which the box-constraints are $\varepsilon$-inactive has nonempty interior. Under this assumption, we can apply the same method in this work to find an element in the new critical cone to approximate the difference of the control and the extremal. By using the strongly active set when estimate the difference of the value functions, we may achieve the aim.

Motivations: First, the second order optimality conditions for periodic optimal control problems governed by semi-linear parabolic equations have not been touched upon, to our best knowledge. However, the periodic optimal control problems are important: from the perspective of applications, the periodic controls can be efficiently applied to some practices (see for example [21] for the chemical reaction process, and [37] for the aircraft model), while from the perspective of mathematics, the period condition is an important kind of state constraint. Second, the second order optimality conditions for non-well-posed optimal control problems are very few. But, the non-well-posed optimal control problems contains many interesting control systems, and the methods dealing with such kind of problems usually differ from those used in the studies of well-posed optimal control problems. These motivate us to study the second order optimality conditions for the problem $(P)$.

Strategy and novelty: Since the problem is non-well-posed, we treat $y$ and $u$ as two independent variables and regard the equation (1.3) as a constraint. Then the non-well-posed problem $(P)$ can be transferred to a 
well-posed optimization problem. This idea was initiated by J.L. Lions (see [30]), and then was used in other references (see, for instance, [23, 40-42]). It seems for us that the current paper is the first one which uses the above idea to study the second order optimality conditions for non-well-posed optimal control problems. Here we would like to mention [11], where the authors obtained the second order optimality conditions for some point-wise state constrained optimal control problem governed by some 1-D parabolic equation with the cubic nonlinearity, via another way: treating the set of all controls making the equation uniquely solvable as the admissible control set, and then getting the optimality conditions by some good properties of the aforementioned admissible control set. In contrast to the method in [11], ours seems to be easier to apply in general.

The periodic state constraint for parabolic equations can be viewed as an equality-type state constraint with infinitely many integral equalities. Indeed, $y(0)=y(T)$ is equivalent to $\int_{\Omega}[y(x, 0)-y(x, T)] e_{j}(x) \mathrm{d} x=0$, $j=1,2, \ldots$ (where $e_{j}, j=1,2, \ldots$, are normalized eigenfunctions of the operator $A$ ). This constraint differs from that in [16] (where the equality-type state constraint with finitely many integral equalities is considered for controlled PDEs) and that in [11] (where the inequality-type state constraint is studied for PDEs). It also differs from the periodic state constraint for controlled ODEs in $[1,9,38,44,46]$, since the periodic state constraint for ODEs contains only finitely many integral equalities. Due to these differences, we need to develop methods to study the second order optimality conditions for our problem $(P):(i)$ In the studies of the second order sufficient optimality condition, we use a kind of controllability for the linearized parabolic equation (see Prop. 3.4), which helps us to overcome the difficulties caused by the state constraint. This idea is partially inspired by [1], where the second order optimality conditions for periodic optimal control problems governed by ODEs was studied. (ii) In the studies of the second order necessary optimality condition, we build up an approximate optimization problem; then derive a second order necessary condition for this approximate problem; finally, by passing to the limit, reach the second order necessary optimality condition for the problem $(P)$. This way was widely used in studies of first order necessary conditions (see, for instance, $[6,39-42]$ ), but seems not to be used to study second order necessary optimality conditions. When use this method in our study, we need a new result: the periodicity restoration property for the linearized parabolic equations under perturbation. (Such kind of restoration problems have been studied in [26, 43], but the results therein are not applicable here.)

Previous works: In the last decades, second order optimality conditions for distributed parameter systems have been extensively studied. We cite here the works $[10,11,13-17,24,25,32,35,36]$. Among them, [10, $14,15,17,32]$ concerned about the second order optimality conditions for nonlinear elliptic optimal control problems, and [11, 13, 24, 25, 35, 36] studied the second order optimality conditions for nonlinear parabolic equations with various inequality type state constraints. The paper [16] considered an abstract optimization problem with finite number of state constraints, which can be applied to elliptic and parabolic optimal control problems with equality/inequality type state constraints with finitely many integral equalities/inequalities.

Second order optimality conditions for periodic optimal control problems of ODEs have been studied in $[1,9,38,44,46]$. The way in most of these works is as: first study the second order variation of the optimal periodic process, then derive a Ricatti-type sufficient optimality condition, with the aid of a accessory problem. Since the periodic state constraint can be viewed as a special case of initial-final state constraint, we mention here also the works $[12,20,33,45]$.

First order optimality conditions for periodic infinite dimensional optimal control problems, including non-well posed problems, have been studied in [4, 5, 27, 39, 40,42], wherein various methods have been developed.

Plan of the work: The rest of this paper is organized as follows: Section 2 presents a first order optimality condition for the weak local minimizer. Section 3 gives the second order sufficient condition. Section 4 shows the second order necessary condition for the weak local minimizer of $(P)$. In Appendix, we give two auxiliary results.

\section{FiRST ORDER OPTIMALITY CONDITION}

Before giving the first order necessary condition for the problem $(P)$, we would like to explain why we do not study the existence of optimal pairs: First, by our understanding, the existence and the optimality conditions for 
optimal pairs are different issues. Second, it is not easy to prove the existence of optimal pairs for the problem $(P)$, since the state equation (1.3) is non-well-posed and the problem involves the periodic state constraint. The non-well-posedness may leads to such a difficulty that for each control, the state might not be uniquely determined (see Thm. 1.5 in $[2]$ ).

Assuming the existence of global minimizer for the problem $(P)$, the first order necessary condition for the minimizer was studied in [42], wherein a type of approximate control problem was introduced in order to deal with the periodic state constraint. We shall use the same idea and method as those used in [42] to obtain the first order optimality condition for a weak local minimizer of the problem $(P)$. The existence of the weak local minimizer is tacitly assumed in this work.

Theorem 2.1. Let $\left(y^{*}, u^{*}\right)$ be a weak local minimizer to the problem $(P)$. Then it is a normal extremal. Moreover, the costate associated with $\left(y^{*}, u^{*}\right)$ is unique.

Proof. Since $\left(y^{*}, u^{*}\right)$ is a weak local minimizer of $(P)$, there is $\delta_{0}>0$, such that $J\left(y^{*}, u^{*}\right) \leq J(y, u), \forall(y, u) \in$ $\mathcal{B}_{\delta_{0}}\left(y^{*}, u^{*}\right)$. Define the set:

$$
\mathcal{P}_{p}^{\delta_{0}} \triangleq\left\{(y, u) \in Y \times L^{2}(Q): y(0)=y(T),\left\|y-y^{*}\right\|_{Y} \leq \delta_{0},\left\|u-u^{*}\right\|_{L^{2}(Q)} \leq \delta_{0}\right\},
$$

wherein the subscript $p$ is used to indicate that the function $y$ is time-periodic. Given $\varepsilon>0$ and $\mu>0$, we define an approximate problem:

$$
\left(P_{\varepsilon, \mu}\right) \quad \text { inf } \quad J_{\varepsilon, \mu}(y, u) \text { over all }(y, u) \in \mathcal{P}_{p}^{\delta_{0}}
$$

Here,

$$
\begin{aligned}
J_{\varepsilon, \mu}(y, u)= & J(y, u)+\frac{\mu}{2} \int_{0}^{T}\left\|u-u^{*}\right\|_{H}^{2} \mathrm{~d} t+\frac{1}{2\left(\hat{r}_{1}+1\right)} \int_{Q}\left|y-y^{*}\right|^{2\left(\hat{r}_{1}+1\right)} \mathrm{d} t \\
& +\frac{1}{2 \varepsilon} \int_{0}^{T}\left\|y^{\prime}(t)+A y(t)+f(t, y)-m u\right\|_{H}^{2} \mathrm{~d} t, y \in Y, u \in L^{2}(Q),
\end{aligned}
$$

where $\hat{r}_{1}=\max \left\{r_{1}, 1\right\}$, with $r_{1}$ given in (1.8). (Notice that $J_{\varepsilon, \mu}(y, u)$ is well defined because of Assumption $(H 1)$ and the fact: $y, y^{*} \in Y$.) We arbitrarily fix $\mu>0$.

Since $\mathcal{P}_{p}^{\delta_{0}} \neq \emptyset$ (which follows from the fact: $\left(y^{*}, u^{*}\right) \in \mathcal{P}_{p}^{\delta_{0}}$ ), by $(2.2)$, as well as $(2.3)$, using the same way as that used in the proof of Lemma 3.1 in [42], we can show that the problem $\left(P_{\varepsilon, \mu}\right)$ has at least one minimizer $\left(y_{\varepsilon}, u_{\varepsilon}\right)$. Then by a very similar method as that used in the proof of Lemma 3.2 in [42], we can obtain that

$$
\left(y_{\varepsilon}, u_{\varepsilon}\right) \rightarrow\left(y^{*}, u^{*}\right) \text { strongly in } Y \times L^{2}(Q) \text {, as } \varepsilon \rightarrow 0 .
$$

By (2.4), we can find $\varepsilon_{0}>0$ so that

$$
\left\|y_{\varepsilon}-y^{*}\right\|_{Y} \leq \delta_{0} / 2 \text { and }\left\|u_{\varepsilon}-u^{*}\right\|_{L^{2}(Q)} \leq \delta_{0} / 2 \text {, when } \varepsilon \in\left(0, \varepsilon_{0}\right) \text {. }
$$

Arbitrarily fix $\varepsilon \in\left(0, \varepsilon_{0}\right)$. Let $\rho \in(0,1)$ and $(z, v) \in \mathcal{Q}_{Y, \delta_{0}} \times \mathcal{Q}_{U, \delta_{0}}$, where

$$
\mathcal{Q}_{Y, \delta_{0}} \triangleq\left\{z \in Y ; z(0)=z(T),\|z\|_{Y} \leq \delta_{0} / 2\right\} ; \quad \mathcal{Q}_{U, \delta_{0}} \triangleq\left\{u \in L^{2}(Q) ;\|u\|_{L^{2}(Q)} \leq \delta_{0} / 2\right\}
$$


Write $y_{\varepsilon}^{\rho} \triangleq y_{\varepsilon}+\rho z$ and $u_{\varepsilon}^{\rho} \triangleq u_{\varepsilon}+\rho v$. Then, by (2.5) and (2.6), we have $\left(y_{\varepsilon}^{\rho}, u_{\varepsilon}^{\rho}\right) \in \mathcal{P}_{p}^{\delta_{0}}$. This, along with the optimality of $\left(y_{\varepsilon}, u_{\varepsilon}\right)$ to $\left(P_{\varepsilon, \mu}\right)$, yields

$$
\frac{J_{\varepsilon}\left(y_{\varepsilon}^{\rho}, u_{\varepsilon}^{\rho}\right)-J_{\varepsilon}\left(y_{\varepsilon}, u_{\varepsilon}\right)}{\rho} \geq 0
$$

By Assumptions (H1)-(H3), we can pass to the limit for $\rho \rightarrow 0$ in the above to get that for any $(z, v) \in$ $\mathcal{Q}_{Y, \delta_{0}} \times \mathcal{Q}_{U, \delta_{0}}$,

$$
\begin{aligned}
0 \leq & \int_{0}^{T}\left(\left\langle g_{y}\left(t, y_{\varepsilon}\right), z\right\rangle_{H}+\left\langle h^{\prime}\left(u_{\varepsilon}\right), v\right\rangle_{H}+\mu\left\langle u_{\varepsilon}-u^{*}, v\right\rangle_{H}\right) \mathrm{d} t \\
& +\int_{0}^{T}\left\langle p_{\varepsilon}, z^{\prime}+A z+f_{y}\left(t, y_{\varepsilon}\right) z-m v\right\rangle_{H} \mathrm{~d} t \\
& +\int_{Q}\left(y_{\varepsilon}-y^{*}\right)^{\left(2 \hat{r}_{1}+1\right)} z \mathrm{~d} x \mathrm{~d} t
\end{aligned}
$$

where $p_{\varepsilon}$ is given by

$$
p_{\varepsilon}=\left[y_{\varepsilon}^{\prime}(t)+A y_{\varepsilon}(t)+f_{y}\left(t, y_{\varepsilon}\right) y_{\varepsilon}(t)\right] / \varepsilon .
$$

By taking $v=0$ in (2.7), we find

$$
\int_{0}^{T}\left[\left\langle g_{y}\left(t, y_{\varepsilon}\right), z\right\rangle_{H}+\left\langle p_{\varepsilon}, \mathcal{A}_{\varepsilon} z\right\rangle_{H}+\left\langle\left(y_{\varepsilon}-y^{*}\right)^{\left(2 \hat{r}_{1}+1\right)}, z\right\rangle_{H}\right] \mathrm{d} t=0,
$$

for all $z \in D\left(\mathcal{A}_{\varepsilon}\right):=\{z \in Y \mid z(0)=z(T)\}$, where $\mathcal{A}_{\varepsilon}: D\left(\mathcal{A}_{\varepsilon}\right) \rightarrow L^{2}(0, T ; H)$ is defined by

$$
\mathcal{A}_{\varepsilon} z=z^{\prime}+A z+f_{y}\left(t, y_{\varepsilon}\right) z, \quad z \in D\left(\mathcal{A}_{\varepsilon}\right) .
$$

Let $\mathcal{A}_{\varepsilon}^{*}$ be the adjoint operator of $\mathcal{A}_{\varepsilon}$. Then we have that $D\left(\mathcal{A}_{\varepsilon}^{*}\right)=\{\varphi \in Y \mid \varphi(0)=\varphi(T)\}$ and

$$
\mathcal{A}_{\varepsilon}^{*} \varphi=-\varphi^{\prime}+A \varphi+f_{y}\left(t, y_{\varepsilon}\right) \varphi, \quad \varphi \in D\left(\mathcal{A}_{\varepsilon}^{*}\right)
$$

Thus, from $(2.8)$, we see that $p_{\varepsilon}$ is in $D\left(\mathcal{A}_{\varepsilon}^{*}\right)$ and satisfies

$$
\left\{\begin{array}{l}
-p_{\varepsilon}^{\prime}(t)+A p_{\varepsilon}(t)+f_{y}\left(t, y_{\varepsilon}\right) p_{\varepsilon}(t)=-g_{y}\left(t, y_{\varepsilon}\right)-\left(y_{\varepsilon}-y^{*}\right)^{2 \hat{r}_{1}+1}, \quad t \in(0, T) \\
p_{\varepsilon}(0)=p_{\varepsilon}(T)
\end{array}\right.
$$

Meanwhile, by taking $z=0$ in (2.7), we see

$$
m p_{\varepsilon}(t)=h^{\prime}\left(u_{\varepsilon}(t)\right)+\mu\left(u_{\varepsilon}(t)-u^{*}(t)\right) \text { for a.e. } t \in(0, T) .
$$

From (2.10) and (2.5), we find that $\left\{p_{\varepsilon}\right\}_{\varepsilon \in\left(0, \varepsilon_{0}\right)}$ is bounded in $L^{2}(Q)$. Then, by the observability of parabolic equations (see [22], Lem. 3.2), we have that $\left\{p_{\varepsilon}(0)\right\}_{\varepsilon \in\left(0, \varepsilon_{0}\right)}$ is bounded in $H$. From this and (2.9), we can easily show that $\left\{p_{\varepsilon}\right\}_{\varepsilon \in\left(0, \varepsilon_{0}\right)}$ is bounded in $L^{2}(0, T ; V) \cap C([0, T] ; H)$. Finally, one can use the same way as that used in the proof of Theorem 3.1 in [42] to prove that

$$
\begin{gathered}
p_{\varepsilon} \rightarrow p^{*}, \text { strongly in } L^{2}(0, T ; V) \cap C([0, T] ; H), \\
\text { weakly in } Y,
\end{gathered}
$$


for some $p^{*} \in Y$.

Now, by (2.4), (2.11) and Assumptions (H1)-(H3), using the same way to that used in the proof of Lemma 3.1 in [42], we can pass to the limit for $\varepsilon \rightarrow 0$ in (2.9) and (2.10) to get (1.6) and (1.7). So $\left(y^{*}, u^{*}\right)$ is a normal extremal.

Finally, by the observability of parabolic equations (see [22], Lem. 3.2 or [42], Cor. 2.1), we can easily see that the costate, associated with $\left(y^{*}, u^{*}\right)$, is unique. This ends the proof of Theorem 2.1.

\section{SeCond order SUfFicient optimality CONDition}

Throughout this section, we let $\left(y^{*}, u^{*}\right)$ be a normal extremal for $(P)$ associated with the costate $p^{*}$. The aim of this section is to give a second-order sufficient condition to ensure that $\left(y^{*}, u^{*}\right)$ is a weak local minimizer. Recall that $\mathcal{L}$ is given by $(1.14)$ with the above $p^{*} ; \mathcal{C}\left(y^{*}, u^{*}\right)$ is given by $(1.16) ; \mathcal{B}_{\varepsilon}\left(y^{*}, u^{*}\right)$ is given by $(1.5)$, where $(\hat{y}, \hat{u})=\left(y^{*}, u^{*}\right)$.

Before giving the second order sufficient optimality conditions, we first present a regularity result of the Lagrangian function defined in (1.14).

Theorem 3.1. Given $(y, u) \in Y \times L^{2}(Q)$, the Lagrangian function $\mathcal{L}$, defined by (1.14), is twice continuously Fréchet differentiable at $(y, u)$ and the second order derivative $\mathcal{L}^{\prime \prime}(y, u) \in L\left(Y \times L^{2}(Q) ; L\left(Y \times L^{2}(Q) ; \mathbb{R}\right)\right)$ is given by

$$
\left[\mathcal{L}^{\prime \prime}(y, u)(\hat{z}, \hat{v})\right](z, v)=\int_{Q}\left[g_{y y}(t, y) \hat{z} z+p^{*} f_{y y}(t, y) \hat{z} z+\alpha \hat{v} v\right] \mathrm{d} x \mathrm{~d} t,(\hat{z}, \hat{v}),(z, v) \in Y \times L^{2}(Q) .
$$

In particular,

$$
\mathcal{L}^{\prime \prime}(y, u)(z, v)^{2}=\int_{Q}\left[g_{y y}(t, y) z^{2}+p^{*} f_{y y}(t, y) w^{2}+\alpha v^{2}\right] \mathrm{d} x \mathrm{~d} t,(z, v) \in Y \times L^{2}(Q),
$$

where $\mathcal{L}^{\prime \prime}(y, u)(z, v)^{2}$ denotes $\left[\mathcal{L}^{\prime \prime}(y, u)(z, v)\right](z, v)$.

The proof of Theorem 3.1 is given in the Appendix. The main result in this section is as follows.

Theorem 3.2. Suppose that $\left(y^{*}, u^{*}\right)$ satisfies (1.15). Then, there are $\varepsilon>0$ and $\sigma>0$ such that

$$
J(y, u)-J\left(y^{*}, u^{*}\right) \geq \sigma\left(\left\|y-y^{*}\right\|_{Y}^{2}+\left\|u-u^{*}\right\|_{L^{2}(Q)}^{2}\right) \text { for all }(y, u) \in \mathcal{B}_{\varepsilon}\left(y^{*}, u^{*}\right) .
$$

Remark 3.3. It follows by $(3.3)$ that $\left(y^{*}, u^{*}\right)$ is a weak local minimizer for $(P)$.

To prove Theorem 3.2, we need the following Proposition 3.4, which is the another version of the null controllability for the linearized equation:

$$
\left\{\begin{array}{l}
z^{\prime}(t)+A z(t)+f_{y}\left(t, y^{*}\right) z(t)=m v(t), t \in(0, T) \\
z(0)=z_{0}
\end{array}\right.
$$

where $v \in L^{2}(Q), z_{0} \in H, y^{*}$ is given by Theorem 3.2, and $m$ is the characteristic function associated to $\omega \subset \Omega$. Notice that (3.4) is well posed (see Thm. A.1 in Appendix). Thus, we can write $z\left(\cdot ; z_{0}, v\right)$ for the unique solution to (3.4) corresponding to $z_{0}$ and $v$.

Proposition 3.4. Given $\xi \in V$, there is $v \in L^{2}(Q)$ such that

$$
z(0 ; \xi, v)=\xi+z(T ; \xi, v)
$$


and

$$
\|z\|_{Y}+\|v\|_{L^{2}(Q)} \leq C\|\xi\|_{V}
$$

where $z(\cdot ; \xi, v)$ is the solution to (3.4) with $z_{0}=\xi$ and where $C>0$ is independent of $\xi$.

Proof. By Assumption (H1) and the Sobolev imbedding Theorem, we get that

$$
\left\|f_{y}\left(t, y^{*}\right)\right\|_{L^{3}(\Omega)} \leq C\left(\int_{\Omega}\left(1+\left|y^{*}\right|^{r_{1}}\right)^{3} \mathrm{~d} x\right)^{\frac{1}{3}} \leq C\left[1+\left(\int_{\Omega}\left|y^{*}\right|^{3 r_{1}} \mathrm{~d} x\right)^{\frac{1}{3}}\right] \leq C\left(1+\left\|y^{*}\right\|_{V}^{r_{1}}\right), t>0 .
$$

Since $y^{*} \in Y \hookrightarrow C([0, T] ; V)$, we can infer that $f_{y}\left(t, y^{*}\right) \in L^{\infty}\left(0, T ; L^{3}(\Omega)\right)$. Then, according to Lemma 3.1 in [22], the equation (3.4) is null controllable. Thus, given $\xi \in V$, there is $v \in L^{2}(Q)$ so that

$$
z(T ; \xi, v)=0
$$

and so that for some $C>0$ independent of $\xi$

$$
\|v\|_{L^{2}(Q)} \leq C\|\xi\|_{V} .
$$

Now, (3.5) follows from (3.7), while (3.6) follows from (3.8) and Theorem A.1 in the Appendix. This ends the proof.

Now, we give the proof of Theorem 3.2.

Proof of Theorem 3.2. Arbitrarily fix $\varepsilon \in(0,1)$, and then arbitrarily fix an admissible pair $(y, u)$ in $\mathcal{B}_{\varepsilon}\left(y^{*}, u^{*}\right)$ which is defined by (1.5). Then, we have

$$
\left\|y-y^{*}\right\|_{Y} \leq \varepsilon \text { and }\left\|u-u^{*}\right\|_{L^{2}(Q)} \leq \varepsilon .
$$

Let

$$
\delta u \triangleq u-u^{*} \text { and } \delta y \triangleq y-y^{*} .
$$

Notice that $(\delta y, \delta u)$ may be not in $\mathcal{C}\left(y^{*}, u^{*}\right)$, thus we cannot directly use (1.15).

The rest of the proof is organized in three steps.

Step 1. We prove that there is $(z, v) \in \mathcal{C}\left(y^{*}, u^{*}\right)$ such that for some $C>0$ (independent of $y, u$ and $\varepsilon$ ) so that

$$
\|(z, v)-(\delta y, \delta u)\|_{Y \times L^{2}(Q)} \leq C\|\delta y\|_{Y}^{2} .
$$

Here and in what follows, $C>0$ is a positive constant (independent of $y, u, \varepsilon$ ) which may varies in different contexts.

First, let $w(\cdot) \triangleq z(\cdot ; \delta y(0), \delta u)$ be the solution to (3.4) with $v=\delta u$ and $z_{0}=\delta y(0)$. We claim that for some $C>0$ independent of $\delta y$,

$$
\|\delta y-w\|_{Y} \leq C\|\delta y\|_{Y}^{2} .
$$

Indeed, if we choose $\phi \triangleq \delta y-w$, then $\phi$ solves

$$
\left\{\begin{array}{l}
\phi^{\prime}(t)+A \phi(t)+f_{y}\left(t, y^{*}\right) \phi(t)+f(t, y)-f\left(t, y^{*}\right)-f_{y}\left(t, y^{*}\right) \delta y=0, \quad t \in(0, T) \\
\phi(0)=0 .
\end{array}\right.
$$


Meanwhile, it follows from the mean value theorem that there is $\xi$ lying between $y^{*}$ and $y$ (That is, for each $(t, x) \in Q$, there is $\theta(t, x) \in(0,1)$ so that $\xi(t, x)=y^{*}(t, x)+\theta(t, x)\left(y(t, x)-y^{*}(t, x)\right)$.) so that

$$
f(t, y)-f\left(t, y^{*}\right)-f_{y}\left(t, y^{*}\right) \delta y=\frac{1}{2} f_{y y}(t, \xi)(\delta y)^{2} \text { for each } t \geq 0 .
$$

This, along with Assumption (H1), the continuous imbedding $V \hookrightarrow L^{q}(\Omega)$ for $q>0$ when $N=1,2,0<q \leq 6$ when $N=3$, and the imbedding $Y \hookrightarrow C([0, T] ; V)$, yields

$$
\begin{aligned}
& \int_{0}^{T} \int_{\Omega}\left|f(t, y)-f\left(t, y^{*}\right)-f_{y}\left(t, y^{*}\right) \delta y\right|^{2} \mathrm{~d} x \mathrm{~d} t \\
= & \frac{1}{4} \int_{0}^{T} \int_{\Omega} f_{y y}^{2}(t, \xi)|\delta y|^{4} \mathrm{~d} x \mathrm{~d} t \\
\leq & C \int_{0}^{T} \int_{\Omega}\left(|\xi|^{2 r_{2}}+1\right)|\delta y|^{4} \mathrm{~d} x \mathrm{~d} t \\
\leq & C \int_{0}^{T}\left(\int_{\Omega}\left(|\xi|^{2 r_{2}}+1\right)^{3} \mathrm{~d} x\right)^{1 / 3}\left(\int_{\Omega}|\delta y|^{6} \mathrm{~d} x\right)^{2 / 3} \mathrm{~d} t \\
\leq & C \int_{0}^{T}\left(\int_{\Omega}\left(\left|y^{*}\right|^{6 r_{2}}+|y|^{6 r_{2}}+1\right) \mathrm{d} x\right)^{1 / 3}\left(\int_{\Omega}|\delta y|^{6} \mathrm{~d} x\right)^{2 / 3} \mathrm{~d} t \\
\leq & C \int_{0}^{T}\left(\left\|y^{*}\right\|_{L^{6 r_{2}}(\Omega)}^{2 r_{2}}+\|y\|_{L^{6 r_{2}(\Omega)}}^{2 r_{2}}+1\right)\|\delta y\|_{L^{6}(\Omega)}^{4} \mathrm{~d} t \\
\leq & C\left(\left\|y^{*}\right\|_{Y}^{2 r_{2}}+\|y\|_{Y}^{2 r_{2}}+1\right)\|\delta y\|_{Y}^{4} \\
\leq & C\left[\left\|y^{*}\right\|_{Y}^{2 r_{2}}+\left(\left\|y^{*}\right\|_{Y}+1\right)^{2 r_{2}}+1\right]\|\delta y\|_{Y}^{4} \leq C\|\delta y\|_{Y}^{4} .
\end{aligned}
$$

Now, by (3.13), Theorem A.1 (in Appendix) and (3.14), we find

$$
\|\phi\|_{Y} \leq C\left\|f(t, y)-f\left(t, y^{*}\right)-f_{y}\left(t, y^{*}\right) \delta y\right\|_{L^{2}(Q)} \leq C\|\delta y\|_{Y}^{2}
$$

which leads to (3.12).

Second, according to Proposition 3.4, there exists $(\tilde{z}, \tilde{v}) \in Y \times L^{2}(Q)$ so that

$$
\left\{\begin{array}{l}
\tilde{z}^{\prime}(t)+A \tilde{z}(t)+f_{y}\left(t, y^{*}\right) \tilde{z}(t)=m \tilde{v}(t), t \in(0, T) \\
\tilde{z}(0)=\tilde{z}(T)+w(T)-w(0)
\end{array}\right.
$$

and

$$
\|(\tilde{z}, \tilde{v})\|_{Y \times L^{2}(Q)} \leq\|w(T)-w(0)\|_{V} .
$$

Let

$$
z \triangleq \tilde{z}+w \text { and } v \triangleq \tilde{v}+\delta u
$$

Then, by (3.16) we have

$$
\|(z, v)-(w, \delta u)\|_{Y \times L^{2}(Q)}=\|(\tilde{z}, \tilde{v})\|_{Y \times L^{2}(Q)} \leq\|w(T)-w(0)\|_{V}
$$


and by (3.4) (with $v=\delta u$ and $\left.z_{0}=\delta y(0)\right)$ and (3.15), we find

$$
\left\{\begin{array}{l}
z^{\prime}(t)+A z(t)+f_{y}\left(t, y^{*}\right) z(t)=m v(t), t \in(0, T) \\
z(0)=z(T)
\end{array}\right.
$$

From (3.19) and (1.16), we see that $(z, v) \in \mathcal{C}\left(y^{*}, u^{*}\right)$. By (3.18), (3.12), and the fact $w(0)=\delta y(0)=\delta y(T)$, we find

$$
\begin{aligned}
& \|(z, v)-(\delta y, \delta u)\|_{Y \times L^{2}(Q)} \\
\leq & \|(z, v)-(w, \delta u)\|_{Y \times L^{2}(Q)}+\|(w, \delta u)-(\delta y, \delta u)\|_{Y \times L^{2}(Q)} \\
\leq & C\left(\|w(T)-w(0)\|_{V}+\|\delta y\|_{Y}^{2}\right) \\
= & C\left(\|w(T)-\delta y(T)\|_{V}+\|\delta y\|_{Y}^{2}\right) .
\end{aligned}
$$

Because $\|w(T)-\delta y(T)\|_{V} \leq C\|w-\delta y\|_{Y}$ (which follows from the fact: $Y \hookrightarrow C([0, T] ; V)$ ), we find from (3.20) and (3.12) that $(z, v)$ given by (3.17) satisfies (3.11).

Hence, we reach the aim of Step 1.

Step 2. We prove

$$
J(y, u)-J\left(y^{*}, u^{*}\right) \geq \frac{1}{2} \mathcal{L}^{\prime \prime}\left(y^{*}, u^{*}\right)(\delta y, \delta u)^{2}-C \varepsilon\|\delta y\|_{Y}^{2} .
$$

Since $(\delta y, \delta u)$ satisfies the equation:

$$
(\delta y)^{\prime}+A \delta y-m \delta u+f(t, y)-f\left(t, y^{*}\right)=0 ; \quad \delta y(0)=\delta y(T)
$$

we see from (1.2) that

$$
\begin{aligned}
& J(y, u)-J\left(y^{*}, u^{*}\right) \\
= & \int_{0}^{T} \int_{\Omega}\left[g(t, y)-g\left(t, y^{*}\right)\right] \mathrm{d} x \mathrm{~d} t+\int_{0}^{T} \int_{\Omega}\left[h(u)-h\left(u^{*}\right)\right] \mathrm{d} x \mathrm{~d} t \\
& +\int_{0}^{T} \int_{\Omega} p^{*}\left[\delta y^{\prime}+A \delta y-m \delta u+f(t, y)-f\left(t, y^{*}\right)\right] \mathrm{d} x \mathrm{~d} t .
\end{aligned}
$$

Meanwhile, by (1.6) and (1.7) (which hold because $\left(y^{*}, u^{*}\right)$ is a normal extremal), we have

$$
\begin{aligned}
& \int_{0}^{T} \int_{\Omega} p^{*}\left[\delta y^{\prime}+A \delta y-m \delta u\right] \mathrm{d} x \mathrm{~d} t \\
= & \int_{0}^{T} \int_{\Omega}\left[\left(-p^{*^{\prime}}(t)+A p^{*}(t)\right) \delta y-m p^{*}(t) \delta u\right] \mathrm{d} x \mathrm{~d} t \\
= & \int_{0}^{T} \int_{\Omega}\left[-p^{*} f_{y}\left(t, y^{*}\right) \delta y-g_{y}\left(t, y^{*}\right) \delta y-h^{\prime}\left(u^{*}\right) \delta u\right] \mathrm{d} x \mathrm{~d} t
\end{aligned}
$$

Substituting (3.23) into (3.22) leads to

$$
J(y, u)-J\left(y^{*}, u^{*}\right)=\int_{0}^{T} \int_{\Omega}\left[g(t, y)-g\left(t, y^{*}\right)-g_{y}\left(t, y^{*}\right) \delta y\right] \mathrm{d} x \mathrm{~d} t
$$




$$
\begin{aligned}
& \quad+\int_{0}^{T} \int_{\Omega}\left[h(u)-h\left(u^{*}\right)-h^{\prime}\left(u^{*}\right) \delta u\right] \mathrm{d} x \mathrm{~d} t \\
& \quad+\int_{0}^{T} \int_{\Omega} p^{*}\left[f(t, y)-f\left(t, y^{*}\right)-f_{y}\left(t, y^{*}\right) \delta y\right] \mathrm{d} x \mathrm{~d} t \\
& \triangleq I_{1}+I_{2}+I_{3} .
\end{aligned}
$$

Here,

$$
\begin{aligned}
& I_{1}=\int_{0}^{T} \int_{\Omega}\left[g(t, y)-g\left(t, y^{*}\right)-g_{y}\left(t, y^{*}\right) \delta y\right] \mathrm{d} x \mathrm{~d} t ; \\
& I_{2}=\int_{0}^{T} \int_{\Omega}\left[h(u)-h\left(u^{*}\right)-h^{\prime}\left(u^{*}\right) \delta u\right] \mathrm{d} x \mathrm{~d} t \\
& I_{3}=\int_{0}^{T} \int_{\Omega} p^{*}\left[f(t, y)-f\left(t, y^{*}\right)-f_{y}\left(t, y^{*}\right) \delta y\right] \mathrm{d} x \mathrm{~d} t .
\end{aligned}
$$

For the term $I_{1}$, we know that there exists $\hat{\xi}$ lying between $y^{*}$ and $y$, such that

$$
I_{1}=\frac{1}{2} \int_{0}^{T} \int_{\Omega} g_{y y}(t, \hat{\xi})(\delta y)^{2} \mathrm{~d} x \mathrm{~d} t
$$

Then, we can use Assumption (H2) to get

$$
\begin{aligned}
I_{1}= & \frac{1}{2} \int_{0}^{T} \int_{\Omega} g_{y y}\left(t, y^{*}\right)(\delta y)^{2} \mathrm{~d} x \mathrm{~d} t+\frac{1}{2} \int_{0}^{T} \int_{\Omega}\left[g_{y y}(t, \hat{\xi})-g_{y y}\left(t, y^{*}\right)\right](\delta y)^{2} \mathrm{~d} x \mathrm{~d} t \\
\geq & \frac{1}{2} \int_{0}^{T} \int_{\Omega} g_{y y}\left(t, y^{*}\right)(\delta y)^{2} \mathrm{~d} x \mathrm{~d} t-C \int_{0}^{T} \int_{\Omega}\left[\left(1+|y|^{m_{3}}+\left|y^{*}\right|^{m_{3}}\right)(\delta y)^{3}\right] \mathrm{d} x \mathrm{~d} t \\
\geq & \frac{1}{2} \int_{0}^{T} \int_{\Omega} g_{y y}\left(t, y^{*}\right)(\delta y)^{2} \mathrm{~d} x \mathrm{~d} t \\
& -C \int_{0}^{T}\left[\int_{\Omega}(\delta y)^{6} \mathrm{~d} x\right]^{1 / 2}\left[\int_{\Omega}\left(1+|y|^{2 m_{3}}+\left|y^{*}\right|^{2 m_{3}}\right) \mathrm{d} x\right]^{1 / 2} \mathrm{~d} t \\
\geq & \frac{1}{2} \int_{0}^{T} \int_{\Omega} g_{y y}\left(t, y^{*}\right)(\delta y)^{2} \mathrm{~d} x \mathrm{~d} t \\
& -C \int_{0}^{T}\|\delta y\|_{L^{6}(\Omega)}^{3}\left(1+\|y\|_{L^{2 m_{3}}(\Omega)}^{m_{2}}+\left\|y^{*}\right\|_{L^{2 m_{3}(\Omega)}}^{m_{3}}\right) \mathrm{d} t \\
\geq & \frac{1}{2} \int_{0}^{T} \int_{\Omega} g_{y y}\left(t, y^{*}\right)(\delta y)^{2} \mathrm{~d} x \mathrm{~d} t-C\left[1+\left(1+\left\|y^{*}\right\|_{Y}\right)^{m_{3}}+\left\|y^{*}\right\|_{Y}^{m_{3}}\right]\|\delta y\|_{Y}^{3} \\
\geq & \frac{1}{2} \int_{0}^{T} \int_{\Omega} g_{y y}\left(t, y^{*}\right)(\delta y)^{2} \mathrm{~d} x \mathrm{~d} t-C \varepsilon\|\delta y\|_{Y}^{2} .
\end{aligned}
$$

For the term $I_{2}$, we can use Assumption (H3) to find

$$
I_{2}=\frac{1}{2} \int_{0}^{T} \int_{\Omega} h^{\prime \prime}\left(u^{*}\right)(\delta u)^{2} \mathrm{~d} x \mathrm{~d} t=\frac{\alpha}{2} \int_{0}^{T} \int_{\Omega}(\delta u)^{2} \mathrm{~d} x \mathrm{~d} t .
$$


For the term $I_{3}$, we can use Assumption (H1) to see

$$
\begin{aligned}
I_{3} \geq & \frac{1}{2} \int_{0}^{T} \int_{\Omega} p^{*} f_{y y}\left(t, y^{*}\right)(\delta y)^{2} \mathrm{~d} x \mathrm{~d} t \\
& -C \int_{0}^{T} \int_{\Omega}\left[p^{*}\left(1+|y|^{r_{3}}+\left|y^{*}\right|^{r_{3}}\right)(\delta y)^{3}\right] \mathrm{d} x \mathrm{~d} t \\
\geq & \frac{1}{2} \int_{0}^{T} \int_{\Omega} p^{*} f_{y y}\left(t, y^{*}\right)(\delta y)^{2} \mathrm{~d} x \mathrm{~d} t-C \varepsilon\|\delta y\|_{Y}^{2} .
\end{aligned}
$$

Now, (3.21) follows from (3.2) and (3.24)-(3.27).

Step 3. We finish the proof.

Let $(z, v)$ be given by Step 1 . We claim for some $C_{1}>0$ (independent of $y, u$ and $\varepsilon$ ),

$$
J(y, u) \geq J\left(y^{*}, u^{*}\right)+C_{1}\left(\|z\|_{Y}^{2}+\|v\|_{L^{2}(Q)}^{2}\right)-C \varepsilon\|\delta y\|_{Y}^{2} .
$$

To this end, several facts are given in order. First, from (3.21) and (3.2), we find

$$
\begin{aligned}
& J(y, u)-J\left(y^{*}, u^{*}\right)-\frac{1}{2} \mathcal{L}^{\prime \prime}\left(y^{*}, u^{*}\right)(z, v)^{2} \\
\geq & \frac{1}{2} \mathcal{L}^{\prime \prime}\left(y^{*}, u^{*}\right)(\delta y, \delta u)^{2}-\frac{1}{2} \mathcal{L}^{\prime \prime}\left(y^{*}, u^{*}\right)(z, v)^{2}-C \varepsilon\|\delta y\|_{Y}^{2} \\
= & \frac{1}{2} \int_{0}^{T} \int_{\Omega}\left\{\left[p^{*} f_{y y}\left(t, y^{*}\right)+g_{y y}\left(t, y^{*}\right)\right]\left((\delta y)^{2}-z^{2}\right)\right. \\
& \left.+\alpha\left((\delta u)^{2}-v^{2}\right)\right\} \mathrm{d} x \mathrm{~d} t-C \varepsilon\|\delta y\|_{Y}^{2} .
\end{aligned}
$$

Second, using Hölder inequality, and by Assumption (H1), we have

$$
\begin{aligned}
& \int_{0}^{T} \int_{\Omega} p^{*} f_{y y}\left(t, y^{*}\right)\left((\delta y)^{2}-z^{2}\right) \mathrm{d} x \mathrm{~d} t \\
\leq & \int_{0}^{T}\left[\left(\int_{\Omega}\left(p^{*}\right)^{2} f_{y y}^{2}\left(t, y^{*}\right) \mathrm{d} x\right)^{\frac{1}{2}}\left(\int_{\Omega}(\delta y+z)^{2}(\delta y-z)^{2} \mathrm{~d} x\right)^{\frac{1}{2}}\right] \mathrm{d} t \\
\leq & C \int_{0}^{T}\left[\left(\int_{\Omega}\left(p^{*}\right)^{6} \mathrm{~d} x\right)^{\frac{1}{6}}\left(\int_{\Omega}\left(\left|y^{*}\right|^{r_{2}}+1\right)^{3} \mathrm{~d} x\right)^{\frac{1}{3}}\left(\int_{\Omega}(\delta y+z)^{4} \mathrm{~d} x\right)^{\frac{1}{4}}\left(\int_{\Omega}(\delta y-z)^{4} \mathrm{~d} x\right)^{\frac{1}{4}}\right] \mathrm{d} t
\end{aligned}
$$

Since $\left(\left|y^{*}\right|^{r_{2}}+1\right)^{3} \leq C_{1}\left(1+\left|y^{*}\right|^{3 r_{2}}\right), V \hookrightarrow L^{q}(\Omega)$ for $q>0$ when $N=1,2$, and $0<q \leq 6$ when $N=3$, and $Y \hookrightarrow C([0, T] ; V)$, we can infer from (3.30) and (3.11) that

$$
\begin{aligned}
& \int_{0}^{T} \int_{\Omega} p^{*} f_{y y}\left(t, y^{*}\right)\left((\delta y)^{2}-z^{2}\right) \mathrm{d} x \mathrm{~d} t \\
\leq & C_{2}\left\|p^{*}\right\|_{Y}\left(\left\|y^{*}\right\|_{Y}^{r_{2}}+1\right)\|\delta y+z\|_{Y}\|\delta y-z\|_{Y} \\
= & C_{2}\left\|p^{*}\right\|_{Y}\left(\left\|y^{*}\right\|_{Y}^{r_{2}}+1\right)\left(\|2 \delta y+z-\delta y\|_{Y}\|\delta y-z\|_{Y}\right. \\
\leq & C_{2}\left\|p^{*}\right\|_{Y}\left(\left\|y^{*}\right\|_{Y}^{r_{2}}+1\right)\left(2\|\delta y\|_{Y}+C\|\delta y\|_{Y}^{2}\right)\|\delta y-z\|_{Y} \\
\leq & C_{3}\left\|p^{*}\right\|_{Y}\left(\left\|y^{*}\right\|_{Y}^{r_{2}}+1\right) \varepsilon\|\delta y-z\|_{Y} \\
\leq & C_{4}\left\|p^{*}\right\|_{Y}\left(\left\|y^{*}\right\|_{Y}^{r_{2}}+1\right) \varepsilon\|\delta y\|_{Y}^{2} \\
= & C \varepsilon\|\delta y\|_{Y}^{2} .
\end{aligned}
$$


In the last equality, we use $C$ to denote the constant $C_{4}\left\|p^{*}\right\|_{Y}\left(\left\|y^{*}\right\|_{Y}^{r_{2}}+1\right)$. Third, by a very similar way to that used in (3.31), we can obtain

$$
\begin{aligned}
& \int_{0}^{T} \int_{\Omega} g_{y y}\left(t, y^{*}\right)\left((\delta y)^{2}-z^{2}\right) \leq C \varepsilon\|\delta y\|_{Y}^{2} \\
& \int_{0}^{T} \int_{\Omega} \alpha\left((\delta u)^{2}-v^{2}\right) \mathrm{d} x \mathrm{~d} t \leq C \varepsilon\|\delta y\|_{Y}^{2} .
\end{aligned}
$$

From (3.29)-(3.33), it follows that

$$
J(y, u)-J\left(y^{*}, u^{*}\right) \geq \frac{1}{2} \mathcal{L}^{\prime \prime}\left(y^{*}, u^{*}\right)(z, v)^{2}-C \varepsilon\|\delta y\|_{Y}^{2}
$$

Since $\left(y^{*}, u^{*}\right)$ satisfies $(1.15)$, we get from (3.34) that

$$
J(y, u) \geq J\left(y^{*}, u^{*}\right)+\frac{1}{2} \gamma\left(\|z(0)\|_{H}^{2}+\|v\|_{L^{2}(Q)}^{2}\right)-C \varepsilon\|\delta y\|_{Y}^{2} .
$$

Because $\|z\|_{Y}^{2}+\|v\|_{L^{2}(Q)}^{2} \leq C\left(\|z(0)\|_{H}^{2}+\|v\|_{L^{2}(Q)}^{2}\right)$ (which follows from Thm. A.1 in Appendix), (3.28) follows from (3.35).

Next, by (3.9) and (3.11), we see that

$$
\|(z, v)-(\delta y, \delta u)\|_{Y \times L^{2}(Q)} \leq C\|\delta y\|_{Y}^{2} \leq C \varepsilon\|\delta y\|_{Y},
$$

from which, we find

$$
\begin{aligned}
& \|(\delta y, \delta u)\|_{Y \times L^{2}(Q)} \\
\leq & \|(z, v)-(\delta y, \delta u)\|_{Y \times L^{2}(Q)}+\|(z, v)\|_{Y \times L^{2}(Q)} \\
\leq & C \varepsilon\|\delta y\|_{Y}+\|(z, v)\|_{Y \times L^{2}(Q)} .
\end{aligned}
$$

Since $\|\delta y\|_{Y} \leq\|(\delta y, \delta u)\|_{Y \times L^{2}(Q)}$, it follows that

$$
\|(\delta y, \delta u)\|_{Y \times L^{2}(Q)}-C \varepsilon\|(\delta y, \delta u)\|_{Y \times L^{2}(Q)} \leq\|(z, v)\|_{Y \times L^{2}(Q)} .
$$

Since the above inequality holds for any $\varepsilon>0$, we can take $\varepsilon \leq \frac{1}{2 C}$ in the last inequality, getting

$$
\|(\delta y, \delta u)\|_{Y \times L^{2}(Q)} \leq C\|(z, v)\|_{Y \times L^{2}(Q)} .
$$

It follows by (3.28) and (3.36) that for some $C_{2}>0$ (independent of $y, u$ and $\varepsilon$ ),

$$
J(y, u) \geq J\left(y^{*}, u^{*}\right)+C_{2}\left(\|\delta y\|_{Y}^{2}+\|\delta u\|_{L^{2}(Q)}^{2}\right)-C \varepsilon\|\delta y\|_{Y}^{2} .
$$

Taking $\varepsilon$ small enough such that $\varepsilon \leq \frac{C_{2}}{2 C}$, we obtain

$$
J(y, u) \geq J\left(y^{*}, u^{*}\right)+\frac{C_{2}}{2}\left(\|\delta y\|_{Y}^{2}+\|\delta u\|_{L^{2}(Q)}^{2}\right),
$$

which leads to (3.3). This ends the proof of Theorem 3.2. 


\section{SECOND ORDER NECESSARY OPTIMALITY CONDITION}

The aim of this section is to give a second-order second order necessary condition for a weak local minimizer of the problem $(\mathrm{P})$.

Theorem 4.1. Any weak local minimizer $\left(y^{*}, u^{*}\right)$ of $(P)$ satisfies (1.17).

Proof. Our strategy is as follows: we first build up an approximate problem as what we did in the proof of Theorem 2.1; then obtain a second-order necessary condition through using the periodicity restoration property (see Thm. A.4 in Appendix) and the first-order optimality condition for the aforementioned approximate problem (see (2.9) and (2.10) in the proof of Thm. 2.1); finally derive a second-order necessary condition for $\left(y^{*}, u^{*}\right)$.

Let $\left(y^{*}, u^{*}\right)$ be a weak local minimizer associated with the costate $p^{*}$. Let $\mathcal{L}$ be given by (1.14) associated with the aforementioned $p^{*}$. We can define, for each $\varepsilon>0$ and each $\mu>0$, the approximate problem $\left(P_{\varepsilon, \mu}\right)$ by (2.2), as well as (2.1) and (2.3). Arbitrarily fix $\mu>0$. We already proved in the proof of Theorem 2.1 the facts: first, $\left(P_{\varepsilon, \mu}\right)$ has a solution $\left(y_{\varepsilon}, u_{\varepsilon}\right) \in \mathcal{P}_{p}^{\delta_{0}} ;\left(\right.$ Here, $\mathcal{P}_{p}^{\delta_{0}}$ is given by (2.1).) second, $\left(y_{\varepsilon}, u_{\varepsilon}\right)$ satisfies $(2.9)$ and $(2.10)$; third, $\left(y_{\varepsilon}, u_{\varepsilon}\right)$ satisfies $(2.5)$. The rest of the proof is organized by two steps.

Step 1. We derive a second-order necessary condition for $\left(y_{\varepsilon}, u_{\varepsilon}\right)$.

Firstly, we claim that

$$
f_{y}\left(t, y_{\varepsilon}\right) \rightarrow f_{y}\left(t, y^{*}\right) \quad \text { strongly in } L^{\infty}\left(0, T ; L^{3}(\Omega)\right), \text { as } \varepsilon \rightarrow 0
$$

Indeed, for each $(t, x) \in Q$, there exists $\tilde{\theta}(t, x) \in(0,1)$, such that

$$
f_{y}\left(t, y_{\varepsilon}(t, x)\right)-f_{y}\left(t, y^{*}(t, x)\right)=f_{y y}(t, \tilde{\xi}(t, x))\left(y_{\varepsilon}(t, x)-y^{*}(t, x)\right),
$$

where

$$
\tilde{\xi}(t, x)=y^{*}(t, x)+\tilde{\theta}(t, x)\left(y_{\varepsilon}(t, x)-y^{*}(t, x)\right) .
$$

Then, using Assumption (H1) and Hölder's inequality, we find

$$
\begin{aligned}
& \left\|f_{y}\left(t, y_{\varepsilon}\right)-f_{y}\left(t, y^{*}\right)\right\|_{L^{3}(\Omega)} \\
\leq & C\left(\int_{\Omega}\left(1+|\tilde{\xi}|^{r_{2}}\right)^{3}\left|y_{\varepsilon}-y^{*}\right|^{3} \mathrm{~d} x\right)^{\frac{1}{3}} \\
\leq & C\left(\int_{\Omega}\left(1+|\tilde{\xi}|^{3 r_{2}}\right)\left|y_{\varepsilon}-y^{*}\right|^{3} \mathrm{~d} x\right)^{\frac{1}{3}} \\
\leq & C\left(\int_{\Omega}\left(1+|\tilde{\xi}|^{6 r_{2}}\right) \mathrm{d} x\right)^{\frac{1}{6}}\left(\int_{\Omega}\left|y_{\varepsilon}-y^{*}\right|^{6} \mathrm{~d} x\right)^{\frac{1}{6}} \\
\leq & C\left[1+\left(\int_{\Omega}\left|y^{*}+\tilde{\theta}\left(y_{\varepsilon}-y^{*}\right)\right|^{6 r_{2}} \mathrm{~d} x\right)^{\frac{1}{6}}\right]\left(\int_{\Omega}\left|y_{\varepsilon}-y^{*}\right|^{6} \mathrm{~d} x\right)^{\frac{1}{6}} \\
\leq & C\left(1+\left\|y^{*}\right\|_{L^{6 r_{2}}(\Omega)}^{r_{2}}+\left\|y_{\varepsilon}-y^{*}\right\|_{L^{6 r_{2}}(\Omega)}^{r_{2}}\right)\left\|_{\varepsilon}-y^{*}\right\|_{L^{6}(\Omega)} \\
\leq & C\left(1+\left\|y^{*}\right\|_{V}^{r_{2}}+\left\|y_{\varepsilon}-y^{*}\right\|_{V}^{r_{2}}\right)\left\|y_{\varepsilon}-y^{*}\right\|_{V}, \quad t \in(0, T) .
\end{aligned}
$$

Since $Y \hookrightarrow C([0, T] ; V)$, we can see from the above inequality that

$$
\left\|f_{y}\left(t, y_{\varepsilon}\right)-f_{y}\left(t, y^{*}\right)\right\|_{L^{\infty}\left(0, T ; L^{3}(\Omega)\right)} \leq C\left(1+\left\|y^{*}\right\|_{Y}^{r_{2}}+\left\|y_{\varepsilon}-y^{*}\right\|_{Y}^{r_{2}}\right)\left\|y_{\varepsilon}-y^{*}\right\|_{Y} .
$$

From (2.4) and (4.3), we are led to (4.1). 
Now, arbitrarily fix $(z, v)$ in the set:

$$
\left\{(\varphi, u) \in \mathcal{C}\left(y^{*}, u^{*}\right) ;\|(\varphi, u)\|_{Y \times L^{2}(Q)} \leq \delta_{0} / 4\right\}
$$

According to (4.1) and Theorem A.4, there is a family $\left\{\left(z_{\varepsilon}, v_{\varepsilon}\right)\right\}_{\varepsilon>0} \subset Y \times L^{2}(Q)$ such that

$$
z_{\varepsilon}^{\prime}(t)+A z_{\varepsilon}(t)+f_{y}\left(t, y_{\varepsilon}\right) z_{\varepsilon}(t)=m v_{\varepsilon}, \quad z_{\varepsilon}(0)=z_{\varepsilon}(T),
$$

and

$$
\left(z_{\varepsilon}, v_{\varepsilon}\right) \rightarrow(z, v) \text { strongly in } Y \times L^{2}(Q) \text { as } \varepsilon \rightarrow 0 \text {. }
$$

Thus, there is $\varepsilon_{1}>0$ such that, when $\varepsilon \in\left(0, \varepsilon_{1}\right)$,

$$
\left\|z_{\varepsilon}-z\right\|_{Y} \leq \delta_{0} / 4 \text { and }\left\|v_{\varepsilon}-v\right\|_{L^{2}(Q)} \leq \delta_{0} / 4
$$

Let

$$
y_{\varepsilon}^{\rho} \triangleq y_{\varepsilon}+\rho z_{\varepsilon} \text { and } u_{\varepsilon}^{\rho} \triangleq u_{\varepsilon}+\rho v_{\varepsilon} \text {, when } \rho \in(0,1), \varepsilon \in\left(0, \varepsilon_{1}\right) \text {. }
$$

By (2.5) and (4.7), we see that

$$
\left(y_{\varepsilon}^{\rho}, u_{\varepsilon}^{\rho}\right) \in \mathcal{P}_{p}^{\delta_{0}}, \text { when } \rho \in(0,1), \varepsilon \in(0, \tilde{\varepsilon}),
$$

where $\tilde{\varepsilon} \triangleq \min \left\{\varepsilon_{0}, \varepsilon_{1}\right\}$ ( $\varepsilon_{0}$ is given in (2.5)). Then, by (4.8), the optimality of $\left(y_{\varepsilon}, u_{\varepsilon}\right),(2.3)$, Taylor expansion of $J_{\varepsilon}$ at $\left(y_{\varepsilon}, u_{\varepsilon}\right),(2.9)$ and $(2.10)$, we can directly check

$$
\begin{aligned}
0 \leq & J_{\varepsilon}\left(y_{\varepsilon}^{\rho}, u_{\varepsilon}^{\rho}\right)-J_{\varepsilon}\left(y_{\varepsilon}, u_{\varepsilon}\right) \\
= & \frac{\rho^{2}}{2} \int_{Q}\left[g_{y y}\left(t, y_{\varepsilon}\right)+p_{\varepsilon} f_{y y}\left(t, y_{\varepsilon}\right)+\left(2 \hat{r}_{1}+1\right)\left(y_{\varepsilon}-y^{*}\right)^{2 \hat{r}_{1}}\right] z_{\varepsilon}^{2} \mathrm{~d} x \mathrm{~d} t \\
& +\frac{\rho^{2}}{2} \int_{Q}(\mu+\alpha) v_{\varepsilon}^{2} \mathrm{~d} x \mathrm{~d} t+r_{\rho}\left(J_{\varepsilon}\right) \text { for all } \rho \in(0,1), \varepsilon \in(0, \tilde{\varepsilon}),
\end{aligned}
$$

where $r_{\rho}\left(J_{\varepsilon}\right)$ is the remainder term given by

$$
\begin{aligned}
& r_{\rho}\left(J_{\varepsilon}\right) \\
= & \int_{Q} r_{\rho}(g) \mathrm{d} x \mathrm{~d} t+\rho^{3} \frac{2 \hat{r}_{1}\left(2 \hat{r}_{1}+1\right)}{3 !} \int_{Q}\left(y_{\varepsilon}+\theta z_{\varepsilon}-y^{*}\right)^{2 \hat{r}_{1}-1} z_{\varepsilon}^{3} \mathrm{~d} x \mathrm{~d} t \\
& +\frac{1}{8 \varepsilon} \rho^{4} \int_{Q} f_{y y}^{2}\left(t, y_{\varepsilon}\right) z_{\varepsilon}^{4} \mathrm{~d} x \mathrm{~d} t+\frac{1}{2 \varepsilon} \int_{Q} f_{y y}\left(t, y_{\varepsilon}\right) z_{\varepsilon}^{2} r_{\rho}(f) \mathrm{d} x \mathrm{~d} t \\
& +\frac{1}{\varepsilon} \int_{Q} p_{\varepsilon} r_{\rho}(f) \mathrm{d} x \mathrm{~d} t+\frac{1}{2 \varepsilon} \int_{Q} r_{\rho}^{2}(f) \mathrm{d} x \mathrm{~d} t,
\end{aligned}
$$

where $\hat{r}_{1}$ is given by $(2.3), \theta \in(0, \rho)$;

$$
r_{\rho}(g)=g\left(t, y_{\varepsilon}^{\rho}\right)-g\left(t, y_{\varepsilon}\right)-\rho g_{y}\left(t, y_{\varepsilon}\right) z_{\varepsilon}-\frac{\rho^{2}}{2} g_{y y}\left(t, y_{\varepsilon}\right) z_{\varepsilon}^{2}
$$


and

$$
r_{\rho}(f)=f\left(t, y_{\varepsilon}^{\rho}\right)-f\left(t, y_{\varepsilon}\right)-\rho f_{y}\left(t, y_{\varepsilon}\right) z_{\varepsilon}-\frac{\rho^{2}}{2} f_{y y}\left(t, y_{\varepsilon}\right) z_{\varepsilon}^{2}
$$

By Assumption (H2), we see that

$$
\begin{aligned}
& \int_{Q} r_{\rho}(g) \mathrm{d} x \mathrm{~d} t \\
\leq & C \rho^{3} \int_{Q}\left(1+\left|y_{\varepsilon}\right|^{m_{3}}+\left|y_{\varepsilon}^{\rho}\right|^{m_{3}}\right) z_{\varepsilon}^{3} \mathrm{~d} x \mathrm{~d} t \\
\leq & C \rho^{3}=o\left(\rho^{2}\right) .
\end{aligned}
$$

Similarly, one can prove the rest terms in $r_{\rho}\left(J_{\varepsilon}\right)$ are all $o\left(\rho^{2}\right)$. Hence, we have

$$
\lim _{\rho \rightarrow 0} r_{\rho}\left(J_{\varepsilon}\right) / \rho^{2}=0 \text { for each } \varepsilon \in(0, \tilde{\varepsilon})
$$

Now, dividing both sides of (4.9) by $\rho^{2} / 2$, and letting $\rho \rightarrow 0$, we get from (4.12) that

$$
\begin{aligned}
& \int_{Q}\left[g_{y y}\left(t, y_{\varepsilon}\right)+p_{\varepsilon} f_{y y}\left(t, y_{\varepsilon}\right)+\left(2 \hat{r}_{1}+1\right)\left(y_{\varepsilon}-y^{*}\right)^{2 \hat{r}_{1}}\right] z_{\varepsilon}^{2} \mathrm{~d} x \mathrm{~d} t \\
& +\int_{Q}(\mu+\alpha) v_{\varepsilon}^{2} \mathrm{~d} x \mathrm{~d} t \geq 0, \text { for each } \varepsilon \in(0, \tilde{\varepsilon}) .
\end{aligned}
$$

Step 2. We derive the second-order necessary condition by passing to the limit.

By Assumption (H2), (2.4) and (4.6), we have

$$
\begin{aligned}
& \int_{Q}\left|g_{y y}\left(t, y_{\varepsilon}\right) z_{\varepsilon}^{2}-g_{y y}\left(t, y^{*}\right) z^{2}\right| \mathrm{d} x \mathrm{~d} t \\
\leq & \int_{Q}\left|g_{y y}\left(t, y_{\varepsilon}\right)-g_{y y}\left(t, y^{*}\right)\right| z_{\varepsilon}^{2} \mathrm{~d} x \mathrm{~d} t+\int_{Q}\left|g_{y y}\left(t, y^{*}\right)\left(z_{\varepsilon}^{2}-z^{2}\right)\right| \mathrm{d} x \mathrm{~d} t \\
\leq & C \int_{Q}\left(1+\left|y_{\varepsilon}\right|^{m_{3}}+\left|y^{*}\right|^{m_{3}}\right)\left|y_{\varepsilon}-y^{*}\right| z_{\varepsilon}^{2} \mathrm{~d} x \mathrm{~d} t \\
& +C \int_{Q}\left(1+\left|y^{*}\right|^{m_{2}}\right)\left|z_{\varepsilon}+z\right|\left|z_{\varepsilon}-z\right| \mathrm{d} x \mathrm{~d} t \\
\leq & C\left(1+\left\|y^{*}\right\|_{Y}^{m_{3}}+\left\|y_{\varepsilon}\right\|_{Y}^{m_{3}}\right)\left\|z_{\varepsilon}\right\|_{Y}^{2}\left\|y_{\varepsilon}-y^{*}\right\|_{Y} \\
& +C\left(1+\left\|y^{*}\right\|_{Y}^{m_{2}}\right)\left(\|z\|_{Y}+\left\|z_{\varepsilon}\right\|_{Y}\right)\left\|z_{\varepsilon}-z\right\|_{Y} \\
\rightarrow & 0, \text { as } \varepsilon \rightarrow 0 .
\end{aligned}
$$

Hence,

$$
\int_{Q} g_{y y}\left(t, y_{\varepsilon}\right) z_{\varepsilon}^{2} \mathrm{~d} x \mathrm{~d} t \rightarrow \int_{Q} g_{y y}\left(t, y^{*}\right) z^{2} \mathrm{~d} x \mathrm{~d} t, \text { as } \varepsilon \rightarrow 0 .
$$


Recalling that $p^{*} \in Y$ and $p_{\varepsilon} \rightarrow p^{*}$ strongly in $C([0, T] ; H)$ (see (2.11)), we can obtain by Assumption (H1), (2.4) and (4.6) that

$$
\begin{aligned}
& \int_{Q}\left|p_{\varepsilon} f_{y y}\left(t, y_{\varepsilon}\right) z_{\varepsilon}^{2}-p^{*} f_{y y}\left(t, y^{*}\right) z^{2}\right| \mathrm{d} x \mathrm{~d} t \\
\leq & \int_{Q}\left|p_{\varepsilon}-p^{*}\right|\left|f_{y y}\left(t, y_{\varepsilon}\right)\right| z_{\varepsilon}^{2} \mathrm{~d} x \mathrm{~d} t+\int_{Q}\left|p^{*} \| f_{y y}\left(t, y_{\varepsilon}\right)-f_{y y}\left(t, y^{*}\right)\right| z_{\varepsilon}^{2} \mathrm{~d} x \mathrm{~d} t \\
& +\int_{Q}\left|p^{*}\left\|f_{y y}\left(t, y^{*}\right)\right\| z_{\varepsilon}^{2}-z^{2}\right| \mathrm{d} x \mathrm{~d} t \\
\leq & C\left(1+\left\|y_{\varepsilon}\right\|_{Y}^{r_{2}}\right)\left\|z_{\varepsilon}\right\|_{Y}^{2}\left\|p_{\varepsilon}-p^{*}\right\|_{C([0, T] ; H)}+C\left(1+\left\|y^{*}\right\|_{Y}^{r_{3}}+\left\|y_{\varepsilon}\right\|_{Y}^{r_{3}}\right)\left\|p^{*}\right\|_{Y}\left\|z_{\varepsilon}\right\|_{Y}^{2}\left\|y_{\varepsilon}-y^{*}\right\|_{Y} \\
& +C\left\|p^{*}\right\|_{Y}\left(1+\left\|y^{*}\right\|_{Y}^{r_{2}}\right)\left(\|z\|_{Y}+\left\|z_{\varepsilon}\right\|_{Y}\right)\left\|z_{\varepsilon}-z\right\|_{Y} \\
\rightarrow & 0, \text { as } \varepsilon \rightarrow 0 .
\end{aligned}
$$

Consequently, we have that

$$
\int_{Q} p_{\varepsilon} f_{y y}\left(t, y_{\varepsilon}\right) z_{\varepsilon}^{2} \mathrm{~d} x \mathrm{~d} t \rightarrow \int_{Q} p^{*} f_{y y}\left(t, y^{*}\right) z^{2} \mathrm{~d} x \mathrm{~d} t, \quad \text { as } \varepsilon \rightarrow 0
$$

Similarly, by (2.4) and (4.6), one can prove that as $\varepsilon \rightarrow 0$,

$$
\begin{aligned}
& \int_{Q}\left(2 \hat{r}_{1}+1\right)\left(y_{\varepsilon}-y^{*}\right)^{2 \hat{r}_{1}} z_{\varepsilon}^{2} \mathrm{~d} x \mathrm{~d} t \rightarrow 0 \\
& \int_{Q}(\mu+\alpha) v_{\varepsilon}^{2} \mathrm{~d} x \mathrm{~d} t \rightarrow \int_{Q}(\mu+\alpha) v^{2} \mathrm{~d} x \mathrm{~d} t .
\end{aligned}
$$

Now, by (4.14), (4.15) and (4.16), we can pass to the limit for $\varepsilon \rightarrow 0$ in (4.13) to get

$$
\int_{Q}\left[g_{y y}\left(t, y^{*}\right)+p^{*} f_{y y}\left(t, y^{*}\right)\right] z^{2} \mathrm{~d} x \mathrm{~d} t+\int_{Q}(\mu+\alpha) v^{2} \mathrm{~d} x \mathrm{~d} t \geq 0
$$

Since the above inequality holds for any $\mu>0$ and any $(z, v)$ in the set (4.4), we can pass to the limit for $\mu \rightarrow 0$ in the (4.17) to get

$$
\int_{Q}\left[g_{y y}\left(t, y^{*}\right)+p^{*} f_{y y}\left(t, y^{*}\right)\right] z^{2} \mathrm{~d} x \mathrm{~d} t+\alpha \int_{Q} v^{2} \mathrm{~d} x \mathrm{~d} t \geq 0
$$

for all $(z, v)$ in the set (4.4). Since $\mathcal{C}\left(y^{*}, u^{*}\right)$ is a cone, we get (1.17) from (3.2) and (4.18). This ends the proof of Theorem 4.1 .

\section{ApPENDix A.}

Theorem A.1. Let $z_{0} \in H, v \in L^{2}(0, T ; H)$ and $a \in L^{\infty}\left(0, T ; L^{3}(\Omega)\right)$. Then, there is a unique $z \in L^{2}(0, T ; V) \cap$ $W^{1,2}\left([0, T] ; V^{*}\right)$ solving the equation:

$$
z^{\prime}(t)+A z(t)+a(t) z(t)=v(t), 0<t<T ; \quad z(0)=z_{0} .
$$

Moreover, the solution $z$ has the following properties: 
(i) If $z_{0} \in V$, then $z$ belongs to $Y$ and satisfies

$$
\|z\|_{Y} \leq e^{C\left(\|a\|_{L^{\infty}\left(0, T ; L^{3}(\Omega)\right)}+1\right)}\left(\left\|z_{0}\right\|_{V}+\|v\|_{L^{2}(0, T ; H)}\right)
$$

for some $C>0$ (independent of $a, z_{0}$ and $v$ ).

(ii) If $z(0)=z(T)+\xi$ for some $\xi \in V$, then $z$ belongs to $Y$ and satisfies

$$
\|z\|_{Y} \leq e^{C\left(\|a\|_{L^{\infty}\left(0, T ; L^{3}(\Omega)\right)}+1\right)}\left(\|z(0)\|_{H}+\|\xi\|_{V}+\|v\|_{L^{2}(0, T ; H)}\right)
$$

for some $C>0$ (independent of $a, z_{0}$ and $v$ ).

Remark A.2. Functions $a$ and $z$ can be treated as functions of $(x, t) \in \Omega \times(0, T)$. In (A.1), $a(t) z(t)$ is defined in the manner: first, we treat it as the function: $(x, t) \rightarrow a(x, t) v(x, t),(x, t) \in \Omega \times(0, T)$; and then we transfer the aforementioned function (of $(x, t))$ into the function of $t$.

Proof. The proofs of the existence and the uniqueness of the solution for more general linear parabolic equation can be found in [29], or [19] (see Chap. 3 in [29] or Sect. 7.1 in [19]). The properties (i)-(ii) can be easily obtained by the way used in the proof of the existence. For the sake of completeness of this paper, we will give detailed proofs.

Existence. We treat $a$ as a function of $(x, t)$ sometimes. Let $\left\{w_{k}\right\}_{k=1}^{\infty}$ be an orthogonal basis of $V$ and also be an orthonormal basis of $H$. (Such $\left\{w_{k}\right\}_{k=1}^{\infty}$ was given in, for instance, Sect. 6.5 in [19]). Arbitrarily fix a positive integer $m$. We first look for a solution $z_{m}$, in the form of

$$
z_{m}(t)=\sum_{k=1}^{m} d_{m}^{k}(t) w_{k}, \quad t \in[0, T]
$$

to the equation:

$$
\left\{\begin{array}{l}
\left\langle z_{m}^{\prime}(t)+A z_{m}(t)+a(t) z_{m}(t), w_{k}\right\rangle_{H}=\left\langle v(t), w_{k}\right\rangle_{H}, t \in[0, T], k=1,2, \cdots, m \\
z_{m}(0)=\sum_{k=1}^{m}\left\langle z_{0}, w_{k}\right\rangle_{H} w_{k} .
\end{array}\right.
$$

This is equivalent to find a solution $\mathbf{d}_{m}(t)=\left(d_{m}^{1}(t), \cdots, d_{m}^{m}(t)\right)^{\top}$ to the system of linear ordinary differential equations:

$$
\left\{\begin{array}{l}
\mathbf{d}_{m}^{\prime}(t)+\mathcal{A}_{m}(t) \mathbf{d}_{m}(t)=\mathbf{f}_{m}(t), t \in[0, T] \\
\mathbf{d}_{m}(0)=\left(\left\langle z_{0}, w_{1}\right\rangle_{H}, \cdots,\left\langle z_{0}, w_{m}\right\rangle_{H}\right)^{\top}
\end{array}\right.
$$

where $\mathcal{A}_{m}(t)$ is the $m \times m$ matrix with elements $\alpha_{i j}(t), i, j=1,2, \cdots, m$, given by

$$
\alpha_{i j}(t)=\int_{\Omega}\left[\sum_{k, l=1}^{N} a_{k l}(x) \frac{\partial w_{i}}{\partial x_{k}} \frac{\partial w_{j}}{\partial x_{l}}+a(x, t) w_{i}(x) w_{j}(x)\right] \mathrm{d} x,
$$

and $\mathbf{f}_{m}(t)=\left(\left\langle v(t), w_{1}\right\rangle_{H}, \cdots,\left\langle v(t), w_{m}\right\rangle_{H}\right)^{\top}$. Since $a \in L^{\infty}\left(0, T ; L^{3}(\Omega)\right), a_{i j} \in C^{2}(\bar{\Omega})$ and $w_{i} \in V, i=1, \cdots, m$, we get from the Sobolev imbedding: $V \hookrightarrow L^{6}(\Omega)$ that

$$
\int_{\Omega}\left|a(x, t) w_{i}(x) w_{j}(x)\right| \mathrm{d} x \leq\|a\|_{L^{\infty}\left(0, T ; L^{3}(\Omega)\right)}\left\|w_{i}\right\|_{L^{6}(\Omega)}\left\|w_{j}\right\|_{H} \text { for a.e. } t \in(0, T)
$$


This yields that $\mathcal{A}_{m}(\cdot) \in L^{\infty}\left(0, T ; \mathbb{R}^{m \times m}\right)$. Meanwhile, we have $\mathbf{f}_{m}(\cdot) \in L^{2}\left(0, T ; \mathbb{R}^{m}\right)$, because $v \in L^{2}(0, T ; H)$. Thus, according to the standard existence theory for ODEs, there is a unique absolutely continuous function $\mathbf{d}_{m}(t)$ satisfying (A.6) for a.e. $t \in[0, T]$. Then, $z_{m}$, defined by (A.4), solves (A.5) for a.e. $t \in[0, T]$.

Next, multiplying the first equation in (A.5) by $d_{m}^{k}$, taking the sum from $k=1$ to $k=m$, and then integrating over $(0, t)$ (with $t>0)$, we obtain that

$$
\left\|z_{m}(t)\right\|_{H}^{2}+\Lambda \int_{0}^{t}\left\|z_{m}(s)\right\|_{V}^{2} \mathrm{~d} s \leq\left\|z_{m}(0)\right\|_{H}^{2}+\int_{0}^{t} \int_{\Omega} v z_{m} \mathrm{~d} x \mathrm{~d} s-\int_{0}^{t} \int_{\Omega} a z_{m}^{2} \mathrm{~d} x \mathrm{~d} s
$$

Meanwhile, using Young's inequality, we see that

$$
\int_{0}^{t} \int_{\Omega}\left|v z_{m}\right| \mathrm{d} x \mathrm{~d} t \leq \Lambda / 4 \int_{0}^{t}\left\|z_{m}(s)\right\|_{V}^{2} \mathrm{~d} t+C(\Lambda)\|v\|_{L^{2}(0, t ; H)}^{2} ;
$$

using the Hölder inequality, Young's inequality, and the imbedding $V \hookrightarrow L^{6}(\Omega)$, we find

$$
\begin{aligned}
\int_{0}^{t} \int_{\Omega}\left|a z_{m}^{2}\right| \mathrm{d} x \mathrm{~d} s & \leq C \int_{0}^{t}\|a(\cdot, t)\|_{L^{3}(\Omega)}\left\|z_{m}\right\|_{L^{6}(\Omega)}\left\|z_{m}\right\|_{L^{2}(\Omega)} \mathrm{d} t \\
& \leq \Lambda / 4 \int_{0}^{t}\left\|z_{m}(s)\right\|_{V}^{2} \mathrm{~d} t+C(\Lambda)\|a\|_{L^{\infty}\left(0, T ; L^{3}(\Omega)\right)}^{2}\left\|z_{m}\right\|_{L^{2}(0, t ; H)}^{2} .
\end{aligned}
$$

(Here, $\Lambda$ is given by (1.1).) With these estimates, we get from (A.7) that, when $t>0$,

$$
\left\|z_{m}(t)\right\|_{H}^{2}+\int_{0}^{T}\left\|z_{m}(s)\right\|_{V}^{2} \mathrm{~d} s \leq C\left(\left\|z_{m}(0)\right\|_{H}^{2}+\|a\|_{L^{\infty}\left(0, T ; L^{3}(\Omega)\right)}^{2} \int_{0}^{t}\left\|z_{m}(s)\right\|_{H}^{2} \mathrm{~d} s+\|v\|_{L^{2}(0, T ; H)}^{2}\right) .
$$

Using Gronwall's inequality in the above inequality, it leads to

$$
\left\|z_{m}(t)\right\|_{H}+\left\|z_{m}\right\|_{L^{2}(0, T ; V)} \leq e^{C\left(\|a\|_{L^{\infty}\left(0, T ; L^{3}(\Omega)\right)}+1\right)}\left(\left\|z_{m}(0)\right\|_{H}+\|v\|_{L^{2}(0, T ; H)}\right), \forall t>0 .
$$

By (A.5) and (A.8), we see

$$
\left\|z_{m}^{\prime}\right\|_{L^{2}\left(0, T ; V^{*}\right)} \leq e^{C\left(\|a\|_{L^{\infty}\left(0, T ; L^{3}(\Omega)\right)}+1\right)}\left(\left\|z_{m}(0)\right\|_{H}+\|v\|_{L^{2}(0, T ; H)}\right), \forall t>0 .
$$

Now, according to (A.8) and (A.9), there is a subsequence of $\left\{z_{m}\right\}$, denoted in the same manner, and a function $z \in L^{2}(0, T ; V) \cap W^{1,2}\left([0, T] ; V^{*}\right)$ such that

$$
\left\{\begin{array}{l}
z_{m} \rightarrow z \text { weakly in } L^{2}(0, T ; V) \\
z_{m}^{\prime} \rightarrow z^{\prime} \text { weakly in } L^{2}\left(0, T ; V^{*}\right)
\end{array}\right.
$$

By (A.10), we can use a standard argument to pass to the limit for $m \rightarrow \infty$ in (A.5) to see that $z$ solves the equation (A.1). (See Page 105 in [29], or Page 380 in [19] for the details).

Uniqueness. We aim to show that the only solution of (A.1) with $v=0, z_{0}=0$ is $z \equiv 0$. For this purpose, we multiply the equation (A.1) by $z$, integrate on $\Omega \times(0, t)$, and then use the same way in the proof of Existence to get $\sup _{t \in[0, T]}\|z(t)\|_{H} \leq 0$, which leads to the uniqueness. 
Proof of $(i)$. We multiply the equation (A.5) by $\frac{d}{\mathrm{~d} t} d_{m}^{k}(t)$, sum $k=1, \ldots, m$, and integrate on $(0, t)$. Then, we can use the similar way used in the proofs of (A.8) and (A.9) to find

$$
\left\|z_{m}(t)\right\|_{V}+\int_{0}^{T}\left\|z_{m}^{\prime}(t)\right\|_{H}^{2} \mathrm{~d} t \leq e^{C\left(\|a\|_{L^{\infty}\left(0, T ; L^{3}(\Omega)\right)}+1\right)}\left(\left\|z_{m}(0)\right\|_{V}+\|v\|_{L^{2}(0, T ; H)}\right), \forall t>0 .
$$

From (A.11), it follows that $z \in L^{\infty}(0, T ; V) \cap W^{1,2}([0, T] ; H)$. This, along with (A.1), yields that $z \in Y$ and that $z$ satisfies (A.2). (See Thm. 5 in Sect. 7.1 in [19] for the details).

Proof of (ii). We multiply (A.1) by $t \frac{\mathrm{d}}{\mathrm{d} t} d_{m}^{k}(t)$, take the sum from $k=1$ to $k=m$, and integrate on $(0, T)$. Then, we obtain by (A.8) that

$$
\left\|z_{m}(T)\right\|_{V} \leq e^{C\left(\|a\|_{L^{\infty}\left(0, T ; L^{3}(\Omega)\right)}+1\right)}\left(\left\|z_{m}(0)\right\|_{H}+\|v\|_{L^{2}(0, T ; H)}\right) .
$$

Passing to the limit for $m \rightarrow \infty$ in the above inequality, we see that $z(T) \in V$ and

$$
\|z(T)\|_{V} \leq e^{C\left(\|a\|_{L^{\infty}\left(0, T ; L^{3}(\Omega)\right)}+1\right)}\left(\|z(0)\|_{H}+\|v\|_{L^{2}(0, T ; H)}\right) .
$$

Since $z(0)=z(T)+\xi$, we have that $z(0) \in V$, and (A.12) leads to

$$
\|z(0)\|_{V} \leq e^{C\left(\|a\|_{L^{\infty}\left(0, T ; L^{3}(\Omega)\right)}+1\right)}\left(\|z(0)\|_{H}+\|v\|_{L^{2}(0, T ; H)}\right)+\|\xi\|_{V} .
$$

This, along with the property (i), shows (A.3).

Therefore, we finish the proof of Theorem A.1.

Remark A.3. For the case that the potential function $a(\cdot, \cdot)$ is bounded from below, some $L^{\infty}$-estimates for some linear parabolic equations were built up in [34], via a semigroup approach and a comparison principle. Therein, the initial controls are taken from $L^{\infty}(Q)$, while distributed controls are taken from a suitable function space which is properly included in $L^{2}(Q)$. The reasons to choose the $L^{\infty}$ estimates in [34] are as follows: First, they dealt with the point-wise state constraint control problem; Second, they studied the Pontryagin maximum principles point-wisely in both time and the space variables. Since the problem and the aims of the current paper differ from those in [34], we do not need the above-mentioned $L^{\infty}$-estimates. More precisely, our state space is $Y$ (which is imbedded in $C([0, T] ; V)$ ) and our state constraint is periodic. These, along with our assumptions on the nonlinear terms, ensure us to get our optimality conditions, which are point-wise in time variable.

Theorem A.4. Suppose that

$$
a_{\varepsilon} \rightarrow a \text { strongly in } L^{\infty}\left(0, T ; L^{3}(\Omega)\right) \text {, as } \varepsilon \rightarrow 0 .
$$

Then, given $(z, v) \in Y \times L^{2}(Q)$ satisfying

$$
z^{\prime}(t)+A z(t)+a(t) z(t)=m v(t), \quad t \in(0, T) ; \quad z(0)=z(T)
$$

there is a family $\left\{\left(z_{\varepsilon}, v_{\varepsilon}\right)\right\}_{\varepsilon>0} \subset Y \times L^{2}(Q)$, with

$$
z_{\varepsilon}^{\prime}(t)+A z_{\varepsilon}(t)+a_{\varepsilon}(t) z_{\varepsilon}(t)=m v_{\varepsilon}(t), \quad t \in(0, T) ; \quad z_{\varepsilon}(0)=z_{\varepsilon}(T)
$$

so that $\left(z_{\varepsilon}, v_{\varepsilon}\right) \rightarrow(z, v)$ in $Y \times L^{2}(Q)$ as $\varepsilon \rightarrow 0$. 
Proof. According to Theorem A.1, given $\varepsilon>0$, the following equation has a unique solution $w_{\varepsilon} \in Y$ :

$$
\left\{\begin{array}{l}
w_{\varepsilon}^{\prime}(t)+A w_{\varepsilon}(t)+a_{\varepsilon}(t) w_{\varepsilon}(t)=m v(t), \quad t \in(0, T) \\
w_{\varepsilon}(0)=z(0)
\end{array}\right.
$$

We first claim that

$$
w_{\varepsilon} \rightarrow z \text { strongly in } Y \text { as } \varepsilon \rightarrow 0 \text {. }
$$

To this end, we let $\hat{w}_{\varepsilon} \triangleq w_{\varepsilon}-z$. By (A.14) and (A.16), we have

$$
\left\{\begin{array}{l}
\hat{w}_{\varepsilon}^{\prime}(t)+A \hat{w}_{\varepsilon}(t)+\delta a_{\varepsilon}(t) \hat{w}_{\varepsilon}(t)=-\delta a_{\varepsilon}(t) z, t \in(0, T) \\
\hat{w}_{\varepsilon}(0)=0
\end{array}\right.
$$

where $\delta a_{\varepsilon}(t) \triangleq\left[a_{\varepsilon}(t)-a(t)\right]$. By (A.18) and Theorem A.1, there is $C>0$, independent on $\varepsilon$, so that

$$
\begin{aligned}
\left\|\hat{w}_{\varepsilon}\right\|_{Y} & \leq e^{C\left(\left\|\delta a_{\varepsilon}\right\|_{L^{\infty}\left(0, T ; L^{3}(\Omega)\right)}+1\right)}\left\|\delta a_{\varepsilon} z\right\|_{L^{2}(0, T ; H)} \\
& \leq e^{C\left(\left\|\delta a_{\varepsilon}\right\|_{L^{\infty}\left(0, T ; L^{3}(\Omega)\right)}+1\right)}\left\|\delta a_{\varepsilon}\right\|_{L^{\infty}\left(0, T ; L^{3}(\Omega)\right)}\|z\|_{L^{2}(0, T ; V)} .
\end{aligned}
$$

This, along with (A.13), leads to (A.17).

Next, by the same argument as that used in the proof of Proposition 3.4, we can find, for each $\varepsilon>0$, $\left(\tilde{z}_{\varepsilon}, \tilde{v}_{\varepsilon}\right) \in Y \times L^{2}(Q)$ so that

$$
\left\{\begin{array}{l}
\tilde{z}_{\varepsilon}^{\prime}(t)+A \tilde{z}_{\varepsilon}(t)+a_{\varepsilon}(t) \tilde{z}_{\varepsilon}(t)=m \tilde{v}_{\varepsilon}(t), \quad t \in(0, T) \\
\tilde{z}_{\varepsilon}(0)=\tilde{z}_{\varepsilon}(T)+w_{\varepsilon}(T)-w_{\varepsilon}(0)
\end{array}\right.
$$

and so that for some $C_{\varepsilon}>0$ (depending on $\varepsilon$ ),

$$
\left\|\left(\tilde{z}_{\varepsilon}, \tilde{v}_{\varepsilon}\right)\right\|_{Y \times L^{2}(Q)} \leq C_{\varepsilon}\left\|w_{\varepsilon}(T)-w_{\varepsilon}(0)\right\|_{V} .
$$

Moreover, by Lemma 3.1 in [22], we see that the above $C_{\varepsilon}$ depends continuously on $\left\|a_{\varepsilon}\right\|_{L^{\infty}\left(0, T ; L^{3}(\Omega)\right)}$. This, along with (A.13), yields that, for a fixed $\hat{\varepsilon}>0$, there is $C>0$, independent of $\varepsilon$, so that $C_{\varepsilon}<C$ for all $\varepsilon \in(0, \hat{\varepsilon})$. This, together with (A.20), leads to

$$
\left\|\left(\tilde{z}_{\varepsilon}, \tilde{v}_{\varepsilon}\right)\right\|_{Y \times L^{2}(Q)} \leq C\left\|w_{\varepsilon}(T)-w_{\varepsilon}(0)\right\|_{V}, \text { when } \varepsilon \in(0, \hat{\varepsilon}) .
$$

Now, we let $\left(z_{\varepsilon}, v_{\varepsilon}\right) \triangleq\left(w_{\varepsilon}, v\right)+\left(\tilde{z}_{\varepsilon}, \tilde{v}_{\varepsilon}\right)$. Then by (A.16) and (A.19), we have that $\left(z_{\varepsilon}, v_{\varepsilon}\right)$ belongs to $Y \times L^{2}(Q)$ and satisfies (A.15). Since $w_{\varepsilon}(T)-w_{\varepsilon}(0)=w_{\varepsilon}(T)-z(0)=w_{\varepsilon}(T)-z(T)$, it follows from (A.21) that

$$
\begin{aligned}
& \left\|\left(z_{\varepsilon}, v_{\varepsilon}\right)-(z, v)\right\|_{Y \times L^{2}(Q)} \\
\leq & \left\|\left(w_{\varepsilon}, v\right)-(z, v)\right\|_{Y \times L^{2}(Q)}+\left\|\left(\tilde{z}_{\varepsilon}, \tilde{v}_{\varepsilon}\right)\right\|_{Y \times L^{2}(Q)} \\
\leq & C\left\|w_{\varepsilon}-z\right\|_{Y}+\left\|w_{\varepsilon}(T)-z(T)\right\|_{V} \\
\leq & C\left\|w_{\varepsilon}-z\right\|_{Y}, \text { when } \varepsilon \in(0, \hat{\varepsilon}) .
\end{aligned}
$$

By (A.17) and (A.22), it follows that $\left(z_{\varepsilon}, v_{\varepsilon}\right) \rightarrow(z, v)$ in $Y \times L^{2}(Q)$. This ends the proof of Theorem A.4.

At last, we give the proof of Theorem 3.1. 
Proof of Theorem 3.1. Arbitrarily fix $(y, u) \in Y \times L^{2}(Q)$. We organize the proof in two steps.

Step 1. We show that $\mathcal{L}$ is Fréchet differentiable at $(y, u)$ and

$$
\mathcal{L}^{\prime}(y, u)(z, v)=\int_{Q}\left[g_{y}(t, y) z+h^{\prime}(u) v+p^{*}\left(z^{\prime}(t)+A z+f_{y}(t, y) z-m v\right)\right] \mathrm{d} x \mathrm{~d} t,(z, v) \in Y \times L^{2}(Q) .
$$

Arbitrarily fix $(z, v) \in Y \times L^{2}(Q)$. According to Taylor's formula, for each $(t, x) \in Q$, there is $\theta_{1}(t, x)$ and $\theta_{2}(t, x) \in(0,1)$ such that

$$
\begin{aligned}
& g(t, y(t, x)+z(t, x))-g(t, y(t, x))=g_{y}(t, y(t, x)) z(t, x)+\frac{1}{2} g_{y y}\left(t, \xi_{1}(t, x)\right) z^{2}(t, x), \\
& f(t, y(t, x)+z(t, x))-f(t, y(t, x))=f_{y}(t, y(t, x)) z(t, x)+\frac{1}{2} f_{y y}\left(t, \xi_{2}(t, x)\right) z^{2}(t, x),
\end{aligned}
$$

where

$$
\xi_{1}(t, x):=y(t, x)+\theta_{1}(t, x) z(t, x), \xi_{2}(t, x):=y(t, x)+\theta_{2}(t, x) z(t, x) .
$$

These, along with (1.14) and the assumption (H3), yields that

$$
\begin{aligned}
\mathcal{L}(y+z, u+v)-\mathcal{L}(y, u)= & \int_{Q}\left[g_{y}(t, y) z+\frac{1}{2} g_{y y}\left(t, \xi_{1}\right) z^{2}+h^{\prime}(u) v+\frac{1}{2} \alpha v^{2}\right. \\
& \left.+p^{*}\left(z^{\prime}(t)+A z+f_{y}(t, y) z-m v\right)+\frac{1}{2} p^{*} f_{y y}\left(t, \xi_{2}\right) z^{2}\right] \mathrm{d} x \mathrm{~d} t .
\end{aligned}
$$

(Here, $\alpha$ is given by $(H 3)$.$) Write$

$$
\Upsilon_{1}(z, v):=\frac{1}{2} \int_{Q}\left[\left(g_{y y}\left(t, \xi_{1}\right)+p^{*} f_{y y}\left(t, \xi_{2}\right)\right) z^{2}+\alpha v^{2}\right] \mathrm{d} x \mathrm{~d} t .
$$

Then, it follows from (A.24) that

$$
\mathcal{L}(y+z, u+v)=\mathcal{L}(y, u)+\int_{Q}\left[g_{y}(t, y) z+h^{\prime}(u) v+p^{*}\left(z^{\prime}(t)+A z+f_{y}(t, y) z-m v\right] \mathrm{d} x \mathrm{~d} t+\Upsilon_{1}(z, v)\right.
$$

We now claim that

$$
\frac{\left|\Upsilon_{1}(z, v)\right|}{\|(z, v)\|_{Y \times L^{2}(Q)}} \rightarrow 0, \text { as }\|(z, v)\|_{Y \times L^{2}(Q)} \rightarrow 0
$$

Indeed, Using Hölder's inequality and Assumptions (H1)-(H3), we have

$$
\begin{aligned}
& \left|\Upsilon_{1}(z, v)\right| \\
\leq & C \int_{Q}\left[\left(1+\left|\xi_{1}\right|^{m_{2}}\right)+\left|p^{*}\right|\left(1+\left|\xi_{2}\right|^{r_{2}}\right)\right] z^{2} \mathrm{~d} x \mathrm{~d} t+\frac{\alpha}{2}\|v\|_{L^{2}(Q)}^{2} \\
\leq & C \int_{0}^{T}\left[\left(\int_{\Omega}\left(1+\left|\xi_{1}\right|^{m_{2}}\right)^{2} \mathrm{~d} x\right)^{\frac{1}{2}}+\left(\int_{\Omega}\left(p^{*}\right)^{4} \mathrm{~d} x\right)^{\frac{1}{4}}\left(\int_{\Omega}\left(1+\left|\xi_{2}\right|^{r_{2}}\right)^{4} \mathrm{~d} x\right)^{\frac{1}{4}}\right]\left(\int_{\Omega} z^{4} \mathrm{~d} x\right)^{\frac{1}{2}} \mathrm{~d} t \\
& +\frac{\alpha}{2}\|v\|_{L^{2}(Q)}^{2} .
\end{aligned}
$$


Here and throughout Step 1, $C$ stands for a positive constant, independent of $(z, v) \in Y$ and $(t, x) \in Q$, which may vary in different lines. Meanwhile, one can directly check

$$
\begin{array}{r}
\left(1+\left|\xi_{1}\right|^{m_{2}}\right)^{2} \leq C\left(1+\left|y+\theta_{1} z\right|^{2 m_{2}}\right) \leq C\left(1+|y|^{2 m_{2}}+|z|^{2 m_{2}}\right), \\
\left(1+\left|\xi_{2}\right|^{r_{2}}\right)^{4} \leq C\left(1+\left|y+\theta_{2} z\right|^{4 r_{2}}\right) \leq C\left(1+|y|^{4 r_{2}}+|z|^{4 r_{2}}\right) \text { for all }(x, t) \in Q .
\end{array}
$$

Write $\tau_{2}:=\max \left\{r_{2}, m_{2}\right\}$. We obtain from (A.27) and (A.28) that

$$
\begin{aligned}
& \left|\Upsilon_{1}(z, v)\right| \\
\leq & C \int_{0}^{T}\left[\left(\int_{\Omega}\left(1+|y|^{2 m_{2}}+|z|^{2 m_{2}}\right) \mathrm{d} x\right)^{\frac{1}{2}}+\left(\int_{\Omega}\left(p^{*}\right)^{4} \mathrm{~d} x\right)^{\frac{1}{4}}\left(\int_{\Omega}\left(1+|y|^{4 r_{2}}+|z|^{4 r_{2}}\right) \mathrm{d} x\right)^{\frac{1}{4}}\right] \\
& \left(\int_{\Omega} z^{4} \mathrm{~d} x\right)^{\frac{1}{2}} \mathrm{~d} t+\frac{\alpha}{2}\|v\|_{L^{2}(Q)}^{2} \\
\leq & C \int_{0}^{T}\left(1+\left\|p^{*}\right\|_{V}\right)\left(1+\|y\|_{V}^{\tau_{2}}+\|z\|_{V}^{\tau_{2}}\right)\|z\|_{V}^{2} d t+\frac{\alpha}{2}\|v\|_{L^{2}(Q)}^{2} \\
\leq & C\left(1+\left\|p^{*}\right\|_{Y}\right)\left(1+\|y\|_{Y}^{\tau_{2}}+\|z\|_{Y}^{\tau_{2}}\right)\|z\|_{Y}^{2}+\frac{\alpha}{2}\|v\|_{L^{2}(Q)}^{2} .
\end{aligned}
$$

In the last two inequalities, we used the imbedding $V \hookrightarrow L^{q}(\Omega)$ for $q>0$ when $N=1,2$, and $0<q \leq 6$ when $N=3$, and the imbedding $Y \hookrightarrow C([0, T] ; V)$ respectively. Thus, (A.26) follows from (A.29) at once. Finally, by (A.25) and (A.26), we see that (A.23) holds.

Step 2. We show (3.1).

Arbitrarily fix $(z, v),(\hat{z}, \hat{v}) \in Y \times L^{2}(Q)$. By (A.23), using the similar way to that in the proof of (A.24), we can find $\lambda_{1}(t, x), \lambda_{2}(t, x) \in(0,1)$, with $(t, x) \in Q$, so that

$$
\mathcal{L}^{\prime}(y+\hat{z}, u+\hat{v})(z, v)=\mathcal{L}^{\prime}(y, u)(z, v)+\int_{Q}\left[g_{y y}(t, y) \hat{z} z+p^{*} f_{y y}(t, y) \hat{z} z+\alpha \hat{v} v\right] \mathrm{d} x \mathrm{~d} t+\left[\Upsilon_{2}(\hat{z}, \hat{v})\right](z, v),
$$

where

$$
\left[\Upsilon_{2}(\hat{z}, \hat{v})\right](z, v)=\int_{Q}\left[\left(g_{y y}\left(t, \eta_{1}\right)-g_{y y}(t, y)\right) \hat{z} z+p^{*}\left(f_{y y}\left(t, \eta_{2}\right)-f_{y y}(t, y)\right) \hat{z} z\right] \mathrm{d} x \mathrm{~d} t
$$

with

$$
\eta_{1}(t, x)=y(t, x)+\lambda_{1}(t, x) \hat{z}(t, x), \eta_{2}(t, x)=y(t, x)+\lambda_{2}(t, x) \hat{z}(t, x), \quad(t, x) \in Q .
$$

Obviously, $\Upsilon_{2}(w, h)$ can be viewed as a linear operator from $Y \times L^{2}(Q)$ to $\mathbb{R}$. Write $\tau_{3}:=\max \left\{m_{3}, r_{3}\right\}$. Using Hölder's inequality and Assumptions (H1) and (H2), we can obtain

$$
\begin{aligned}
& \left|\left[\Upsilon_{2}(\hat{z}, \hat{v})\right](z, v)\right| \\
\leq & C \int_{Q}\left[\left(1+\left|\eta_{1}\right|^{m_{3}}+|y|^{m_{3}}\right)+\left(1+\left|\eta_{2}\right|^{r_{3}}+|y|^{r_{3}}\right)\right]|z| \hat{z}^{2} \mathrm{~d} x \mathrm{~d} t \\
\leq & \left.C \int_{0}^{T}\left[\left(\int_{\Omega}\left(1+\left|\eta_{1}\right|^{m_{3}}+|y|^{m_{3}}\right)^{2} \mathrm{~d} x\right)^{\frac{1}{2}}+\left(\int_{\Omega}\left(p^{*}\right)^{4} \mathrm{~d} x\right)^{\frac{1}{4}}\left(\int_{\Omega}\left(1+\left|\eta_{2}\right|^{r_{3}}+|y|^{r_{3}}\right)\right)^{4} \mathrm{~d} x\right)^{\frac{1}{4}}\right]\left(\int_{\Omega} z^{2} \hat{z}^{4} \mathrm{~d} x\right)^{\frac{1}{2}} \mathrm{~d} t \\
\leq & C \int_{0}^{T}\left[\left(\int_{\Omega}\left(1+|y|^{2 m_{3}}+|z|^{2 m_{3}}\right) \mathrm{d} x\right)^{\frac{1}{2}}\right.
\end{aligned}
$$




$$
\begin{aligned}
& \left.+\left(\int_{\Omega}\left(p^{*}\right)^{4} \mathrm{~d} x\right)^{\frac{1}{4}}\left(\int_{\Omega}\left(1+|y|^{4 r_{3}}+|z|^{4 r_{3}}\right) \mathrm{d} x\right)^{\frac{1}{4}}\right]\left(\int_{\Omega} \hat{z}^{6} \mathrm{~d} x\right)^{\frac{1}{3}}\left(\int_{\Omega} z^{6} \mathrm{~d} x\right)^{\frac{1}{6}} \mathrm{~d} t \\
\leq & C \int_{0}^{T}\left(1+\left\|p^{*}\right\|_{V}\right)\left(1+\|y\|_{V}^{\tau_{3}}+\|z\|_{V}^{\tau_{3}}\right)\|\hat{z}\|_{V}^{2}\|z\|_{V} \mathrm{~d} t \\
\leq & C\left(1+\left\|p^{*}\right\|_{Y}\right)\left(1+\|y\|_{Y}^{\tau_{3}}+\|z\|_{Y}^{\tau_{3}}\right)\|\hat{z}\|_{Y}^{2}\|z\|_{Y} .
\end{aligned}
$$

Here and throughout Step 2, $C$ stands for a positive constant, independent of $(z, v),(\hat{z}, \hat{v}) \in Y \times L^{2}(Q)$, which may vary in different lines. By (A.31), we see that $\Upsilon_{2}(\hat{z}, \hat{v}) \in L\left(Y \times L^{2}(Q) ; \mathbb{R}\right)$ and,

$$
\left\|\Upsilon_{2}(\hat{z}, \hat{v})\right\|_{L\left(Y \times L^{2}(Q) ; \mathbb{R}\right)} \leq C\left(1+\left\|p^{*}\right\|_{Y}\right)\left(1+\|y\|_{Y}^{\tau_{3}}+\|z\|_{Y}^{\tau_{3}}\right)\|\hat{z}\|_{Y}^{2}
$$

and

$$
\frac{\left\|\Upsilon_{2}(\hat{z}, \hat{v})\right\|_{L\left(Y \times L^{2}(Q) ; \mathbb{R}\right)}}{\|(\hat{z}, \hat{v})\|_{Y \times L^{2}(Q)}} \rightarrow 0, \text { as }\|(\hat{z}, \hat{v})\|_{Y \times L^{2}(Q)} \rightarrow 0
$$

By (A.32) and (A.30), we are led to (3.1).

Step 3. We finish the proof.

First, (3.2) is a direct consequence of (3.1). Second, the continuity of the mapping $(y, u) \rightarrow \mathcal{L}^{\prime \prime}(y, u)$ can be directly derived from (3.1), through using the similar arguments used in Step 2. Indeed, when $\left(y_{1}, u_{1}\right),\left(y_{2}, u_{2}\right) \in$ $Y \times L^{2}(Q)$, we can obtain that

$$
\left\|\mathcal{L}^{\prime \prime}\left(y_{1}, u_{1}\right)-\mathcal{L}^{\prime \prime}\left(y_{2}, u_{2}\right)\right\|_{L\left(Y \times L^{2}(Q) ; L\left(Y \times L^{2}(Q) ; \mathbb{R}\right)\right)} \leq C\left(1+\left\|p^{*}\right\|_{Y}\right)\left(1+\|y\|_{Y}^{\tau_{3}}+\|z\|_{Y}^{\tau_{3}}\right)\left\|y_{1}-y_{2}\right\|_{Y} .
$$

Hence, we complete the proof of Theorem 3.1.

Acknowledgements. The authors want to thank the anonymous referees for their valuable remarks and corrections on the original version of this paper.

\section{REFERENCES}

[1] J. Allwright and R. Vinter, Second order conditions for periodic optimal control problems. Control Cybern. 34 (2005) 617-643.

[2] H. Amann, Periodic solutions of semilinear parabolic equations. In Nonlinear analysis. Elsevier (1978) 1-29.

[3] J.M. Ball, Remarks on blow-up and nonexistence theorems for nonlinear evolution equations. Quart. J. Math. Oxford Ser. (2) 28 (1977) 473-486.

[4] V. Barbu and N.H. Pavel, Optimal control problems with two-point boundary conditions. J. Optim. Theory Appl. 77 (1993) $51-78$.

[5] V. Barbu and N.H. Pavel, Periodic optimal control in Hilbert space. Appl. Math. Optim. 33 (1996) 169-188.

[6] V. Barbu, Analysis and control of nonlinear infinite-dimensional systems. Vol. 190 of Mathematics in Science and Engineering. Academic Press, Inc., Boston, MA (1993).

[7] T. Bayen, J. Frederic Bonnans and F. Silva, Characterization of local quadratic growth for strong minima in the optimal control of semi-linear elliptic equations. Trans. Am. Math. Soc. 366 (2014) 2063-2087.

[8] T. Bayen and F.J. Silva, Second order analysis for strong solutions in the optimal control of parabolic equations. SIAM J. Control Optim. 54 (2016) 819-844.

[9] S. Bittanti, A. Locatelli and C. Maffezzoni, Second-variation methods in periodic optimization. J. Optim. Theory Appl. 14 (1974) 31-49.

[10] J.F. Bonnans, Second-order analysis for control constrained optimal control problems of semilinear elliptic systems. Appl. Math. Optim. 38 (1998) 303-325.

[11] J.F. Bonnans and P. Jaisson, Optimal control of a parabolic equation with time-dependent state constraints. SIAM J. Control Optim. 48 (2010) 4550-4571.

[12] J.F. Bonnans and N.P. Osmolovskiı̆, Second-order analysis of optimal control problems with control and initial-final state constraints. J. Convex Anal. 17 (2010) 885-913. 
[13] E. Casas, J.C. de los Reyes and F. Tröltzsch, Sufficient second-order optimality conditions for semilinear control problems with pointwise state constraints. SIAM J. Optim. 19 (2008) 616-643.

[14] E. Casas and M. Mateos, Second order optimality conditions for semilinear elliptic control problems with finitely many state constraints. SIAM J. Control Optim. 40 (2002) 1431-1454.

[15] E. Casas and F. Tröltzsch, Second-order necessary optimality conditions for some state-constrained control problems of semilinear elliptic equations. Appl. Math. Optim. 39 (1999) 211-227.

[16] E. Casas and F. Tröltzsch, Second-order necessary and sufficient optimality conditions for optimization problems and applications to control theory. SIAM J. Optim. 13 (2002) 406-431.

[17] E. Casas, F. Tröltzsch and A. Unger, Second order sufficient optimality conditions for some state-constrained control problems of semilinear elliptic equations. SIAM J. Control Optim. 38 (2000) 1369-1391.

[18] E. Casas and F. Tröltzsch, Second-order optimality conditions for weak and strong local solutions of parabolic optimal control problems. Viet. J. Math. 44 (2016) 181-202.

[19] L.C. Evans, Partial differential equations. Vol. 19 of Graduate Studies in Mathematics. American Mathematical Society, Providence, RI, second edition (2010).

[20] H. Frankowska, The maximum principle for an optimal solution to a differential inclusion with end points constraints. SIAM J. Control Optim. 25 (1987) 145-157.

[21] F.J.M. Horn and R.C. Lin, Periodic processes: a variational approach. Ind. Eng. Chem. Process Des. Dev. 6 (1967) 221-230.

[22] O. Yu. Imanuvilov and M. Yamamoto, Carleman inequalities for parabolic equations in Sobolev spaces of negative order and exact controllability for semilinear parabolic equations. Publ. Res. Inst. Math. Sci. 39 (2003) 227-274.

[23] K. Ito and K. Kunisch, Augmented Lagrangian-SQP methods for nonlinear optimal control problems of tracking type. SIAM J. Control Optim. 34 (1996) 874-891.

[24] B.T. Kien, V.H. Nhu and A. Rösch, Second-order necessary optimality conditions for a class of optimal control problems governed by partial differential equations with pure state constraints. J. Optim. Theory Appl. 165 (2015) 30-61.

[25] K. Krumbiegel and J. Rehberg, Second order sufficient optimality conditions for parabolic optimal control problems with pointwise state constraints. SIAM J. Control Optim. 51 (2013) 304-331.

[26] L. Lei, Restoration of periodicity for a periodic parabolic system under perturbations in the system conductivity. J. Optim. Theory Appl. 150 (2011) 580-598.

[27] X. Li and J. Yong, Necessary conditions for optimal control of distributed parameter systems. SIAM J. Control Optim. 29 (1991) 895-908.

[28] P. Lin and G. Wang, Some properties for blowup parabolic equations and their application. J. Math. Pures Appl. (9) 101 (2014) 223-255.

[29] J.-L. Lions, Optimal control of systems governed by partial differential equations. Translated from the French by S. K. Mitter. Die Grundlehren der mathematischen Wissenschaften, Band 170. Springer-Verlag, New York-Berlin (1971).

[30] J.-L. Lions, Some methods in the mathematical analysis of systems and their control. Kexue Chubanshe (Science Press), Beijing; Gordon \& Breach Science Publishers, New York (1981).

[31] J. Louis Lions and E. Magenes, Vol. 1 of Non-homogeneous boundary value problems and applications. Springer Science \& Business Media (2012).

[32] H. Lou and J. Yong, Second-order necessary conditions for optimal control of semilinear elliptic equations with leading term containing controls. Math. Control Relat. Fields 8 (2018) 57-88.

[33] H. Maurer and S. Pickenhain, Second-order sufficient conditions for control problems with mixed control-state constraints. $J$. Optim. Theory Appl. 86 (1995) 649-667.

[34] J. Pierre Raymond and H. Zidani, Hamiltonian pontryagin's principles for control problems governed by semilinear parabolic equations. Appl. Math. Optim. 39 (1999) 143-177.

[35] J.-P. Raymond and F. Tröltzsch, Second order sufficient optimality conditions for nonlinear parabolic control problems with state constraints. Discrete Contin. Dynam. Syst. 6 (2000) 431-450.

[36] A. Rösch and F. Tröltzsch, Sufficient second-order optimality conditions for a parabolic optimal control problem with pointwise control-state constraints. SIAM J. Control Optim. 42 (2003) 138-154.

[37] J.L. Speyer, Nonoptimality of the steady-state cruise for aircraft. Aiaa J. 14 (1976) 1604-1610.

[38] J.L. Speyer and R.T. Evans, A second variational theory for optimal periodic processes. IEEE Trans. Automat. Control 29 (1984) 138-148.

[39] G. Wang, Optimal control of parabolic differential equations with two point boundary state constraints. SIAM J. Control Optim. 38 (2000) 1639-1654.

[40] G. Wang, Optimal controls of 3-dimensional Navier-Stokes equations with state constraints. SIAM J. Control Optim. 41 (2002) 583-606.

[41] G. Wang and L. Wang, State constrained optimal control governed by non-well-posed parabolic differential equations. SIAM J. Control Optim. 40 (2002) 1517-1539.

[42] G. Wang and L. Wang, The Carleman inequality and its application to periodic optimal control governed by semilinear parabolic differential equations. J. Optim. Theory Appl. 118 (2003) 429-461.

[43] G. Wang and G. Zheng, The optimal control to restore the periodic property of a linear evolution system with small perturbation. Discrete Contin. Dyn. Syst. Ser. B 14 (2010) 1621-1639.

[44] Q. Wang and J.L. Speyer, Necessary and sufficient conditions for local optimality of a periodic process. SIAM J. Control Optim. 28 (1990) 482-497. 
[45] V. Zeidan, New second-order optimality conditions for variational problems with $C^{2}$-Hamiltonians. SIAM J. Control Optim. 40 (2001) 577-609.

[46] V. Zeidan and P.L. Zezza, Coupled points in the calculus of variations and applications to periodic problems. Trans. Amer. Math. Soc. 315 (1989) 323-335. 